\title{
THE DISTRIBUTION OF HEAT IN THE CYLINDER OF A GAS-ENGINE.
}

By Professor A. H. GIBSON, D.SC, Member, of Untverstty College, DUNDee, aNd W. J. WALKER, B.Sc. (Carnegie Research Scholar), of the Municipat School of Technology, Manchester.

The investigation outlined in this Paper is to some extent the outcome of a suggestion made by the British Association Committee for the investigation of Gaseous Explosions in their report for 1912. The report states (inter alia) :-

... . "The effect of heat flow upon economy is not very marked, and it is. therefore not surprising that no decisive verdict has yet been pronounced on the relation between economy and speed. There is no doubt that, given satisfactory ignition, economy is somewbat improved by increasing the speed, but the relation between speed and economy has not been so precisely determined as to permit a conclusion to be drawn about the part played by turbulence, nor, in view of the complication of the question, does it seem likely that much information can be derived from this source. A more promising line of inquiry would be a direct measurement of jacket losses at different speeds. The Committee are not aware that any accurate measurements of jacket loss at different speeds have ever been undertaken. From some rather rough measurements of this character, it appears that the heat loss per cycle does diminish with increase of speed, but not in proportion thereto."

[The I.Mесн.E.] 
An experimental gas-engine recently installed in the Engineering Laboratories at University College, Dundee, appeared to afford exceptional facilities for such an investigation. This engine, built by the National Gas Engine Co., Ltd., has a cylinder diameter of 11 inches and a stroke of 19 inches. The connecting-rod may be lengthened so as to vary the compression ratio between the limits

FIG. 1.-General Arrangement of Jackets for Exhaust-Pipe and Cylinder, Exhaust-Cooler and Thermometers.
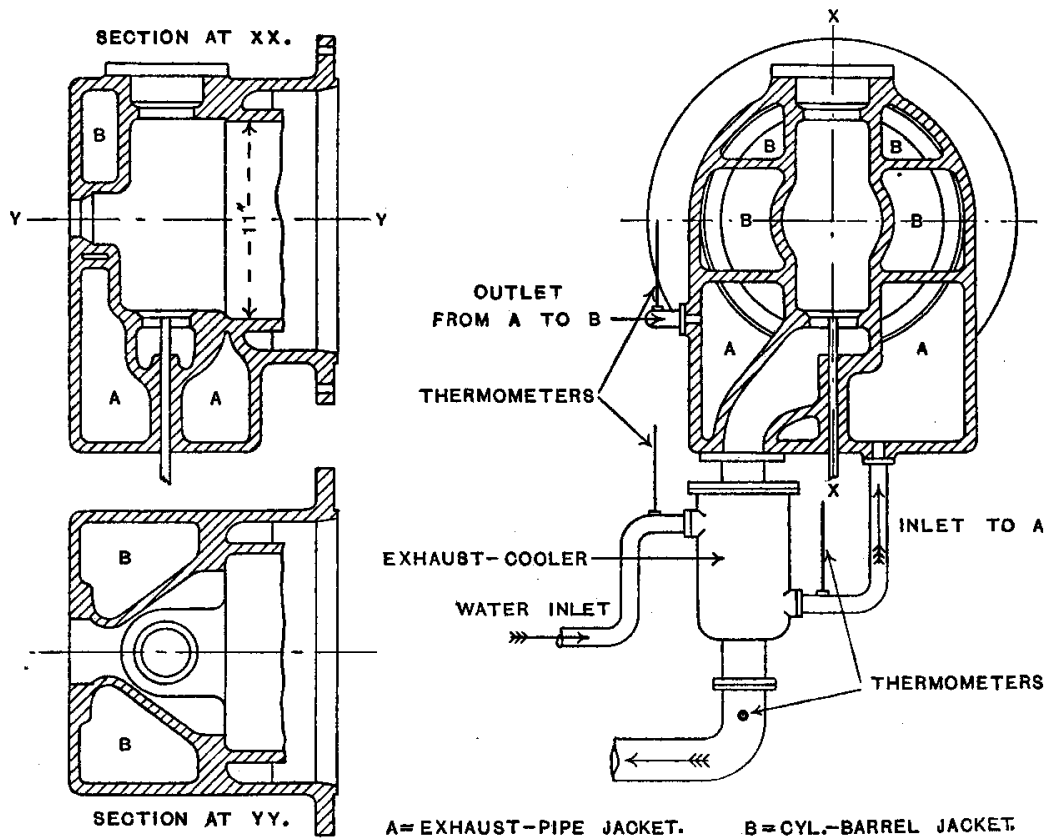

$5 \cdot 17$ and $6 \cdot 62$. Governing is on the hit-and-miss principle. A special feature of the engine consists in the arrangement of the cylinder-jackets, which were specially designed for experimental work. The jacket as usually constructed is here divided into two separate parts. One of these surrounds the exhaust-valve, and that portion of the exhaust passage included within the cylindercasting, while the other covers the breech-end and barrel of the 
cylinder. The jacket water is led in serias through the two sections, its temperature being measured before and after passing through each of these. As is well known, the heat attributed to jacket losses in a gas-engine having the usual arrangement of jackets includes a certain proportion which, correctly, should be attributed to exhaust losses. In the engine under consideration, the magnitude of these two sources of loss can be ascertained with a much higher degree of accuracy. The arrangement of the

Fig. 2.-Exhaust-Gas Cooler.

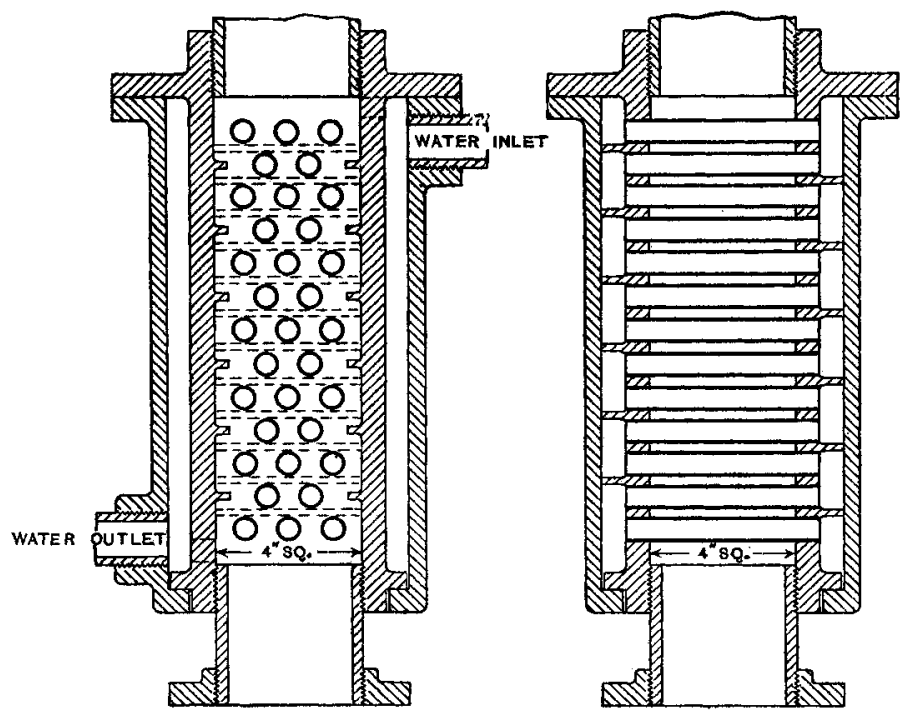

jackets is indicated by the sectional drawing of the breech-end of the cylinder, shown in Fig. 1.

In order to measure the heat contained in the exhaust gases after leaving the cylinder, an exhaust-cooler, Fig. 2, was fitted to the exhaust branch. In this cooler the temperature of the gases is reduced by their passage over a series of 33 tubes, each $\frac{5}{8}$ inch outside diameter and 4 inches long. The jacket water passes through these tubes on its way to the cylinder jackets, and its temperature is measured before and after passing the cooler. 
The trials, as finally carried out, were extended with a view to determine how the distribution of heat through the engine varies with--

(1) The speed of the engine.

(2) The brake horse-power.

(3) The compression ratio.

(4) The richness of the $\frac{\text { air }}{\text { gas }}$ mixture.

The normal speed of the engine is 200 revolutions per minute. In the trials, a range of speeds from 140 to 260 revolutions per minute was examined.

The maximum b.h.p. of the engine depends on the speed and mixture of air and gas. Its values are very approximately as follows :-

\begin{tabular}{|c|c|c|c|}
\hline \multirow{2}{*}{ Ratio $\frac{\mid 3}{\text { Air }}$ Gas (vols.). } & \multicolumn{3}{|c|}{ Speed r.p.m. } \\
\cline { 2 - 4 } & 150 & 200 & 250 \\
\hline 7 & $25 \cdot 0$ & $31 \cdot 5$ & $36 \cdot 0$ \\
9 & $20 \cdot 0$ & $25 \cdot 0$ & $28 \cdot 5$ \\
11 & 16.5 & $20 \cdot 5$ & $23 \cdot 5$ \\
\hline
\end{tabular}

In the trials the b.h.p. was varied from zero up to this full load capacity. Three different compression ratios were adopted. These are respectively $5 \cdot 17,5 \cdot 70$, and $6 \cdot 62$. Three different $\frac{\text { air }}{\text { gas }}$ mixtures were used. In round numbers these are $7: 1,9: 1$, and $11: 1$. In individual trials of the same series the richness of the mixture varied by not more than 5 per cent. on each side of the mean. In the great majority of cases the variation did not exceed 2 per cent. on each side of the mean.

In all some 130 trials were carried out.

Experimental Measurements.-The methods adopted in measuring and calculating the various heat quantities involved, agree in 
principle with those outlined in the Inst. C.E. report on InternalCombustion Engines.*

Gas Supply.-Town gas was used in the engine. An average analysis of this gave the following result:-

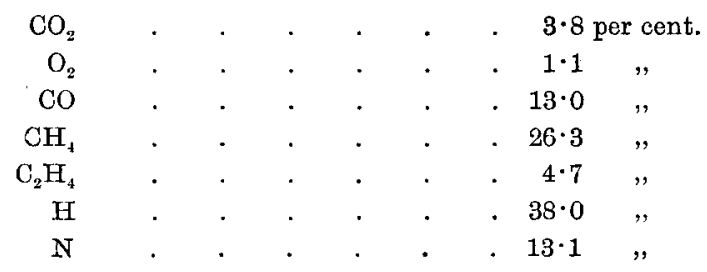

The corresponding lower calorific value (calculated) at standard temperature and pressure is 521.4 B.Th.U. per cubic foot. The gas supply is measured by a dry meter, which was calibrated before the trials against a standard gasometer. The calorific values, higher and lower, were measured by a Boys' calorimeter deriving its supply from the gas-main. The calorimeter was in operation during the whole duration of each trial, and the mean calorific value adopted for each trial is the mean of the results obtained from the calorimeter during this period. The mean of the lower and higher values obtained during the trials were respectively 520 B.Th.U. and 577 B.Th.U. per cubic foot. The lower value has been used in all calculations.

Air Supply.-This is drawn through two 1,500-light dry meters in parallel. These meters were calibrated against a standard gasometer.

Measurement of Work.-The brake horse-power is measured by a water-cooled Prony brake whose drum is mounted alongside the fly-wheel. The indicated horse-power is deduced from diagrams taken with a Dobbie-McInnes gas-engine indicator. $A_{\frac{3}{5} \sigma}$ spring was used for the power diagrams, and a $\frac{1}{16}$ spring for diagrams

* Proceedings, Inst. C.E., 1906, vol. clxiii, page 241. 
intended for the measurement of suction pressures. While no high degree of acouracy is to be anticipated for these measurements of indicated work, they appear to be very consistent.

Jacket Water.-As already stated, the jacket water passes first through the exhaust-gas cooler, then through the jacket surrounding the exhaust valve and exhaust passage and finally through the barrel and breech jacket. On leaving the latter jacket it is measured in one of a pair of calibrated measuring tanks. Temperatures are measured before and after each jacket or section of a jacket, and from a knowledge of the weight of water passing, the heat flow through the walls of each stage is readily computed. The weight of water in each trial was adjusted so as to give a final temperature as near as possible to $145^{\circ} \mathrm{F} .\left(63^{\circ} \mathrm{C}\right)$. In some trials when the temperature had become steady, this final temperature was two or three degrees above or below $145^{\circ} \mathrm{F}$. Sensibly, however, the final temperature was constant over the whole series of trials.

Thermometers.-All thermometers were calibrated before the trials.

Heat in Exhaust Gases.-Systematic analyses of the exhaust gases were carried out, mainly with a view to ensuring that combustion was complete before the end of expansion. In no case was more than a trace of combustible found in the gas. In the great majority of cases no such trace was found.

Of the total heat in the exhaust gases leaving the cylinder, part is absorbed by the water in the exhaust-valve jacket, and part in the exhaust-gas cooler. The cooler is not sufficiently large to cool down the gases to atmospheric temperature, and their temperature on leaving the cooler is usually between $200^{\circ}$ and $300^{\circ} \mathrm{F}$. The heat carried away by these gases is estimated from a knowledge of their weight, specific heat, and temperature. Their weight is known, since it is equal to the weight of air + gas + moisture entering the cylinder. The amount of steam formed during combustion is known from the records of the gas-calorimeter, 
while the specific heat of the dry exhaust gas, which varies a little with its composition, is sensibly equal to $0 \cdot 24$. The mean temperature is measured by means of a mercury thermometer.

Method of Conducting Trials.-Having started up the engine, the governor is adjusted to give the desired speed, and, by reference to the air and gas meters, the air and gas inlet-valves are adjusted until the required air-gas mixture is attained. The necessary brake load is then applied; the ignition is advanced or retarded until a satisfactory diagram with a vertical explosion line is obtained; and the jaoket water is regulated so as to give the required outlet temperature. When all conditions are steady, the trial commences. Each trial extended over some 30 to 45 minutes.

Experimental Data and Results.--In view of the very large number of trials involved, it has been thought inadvisable to attempt to give the detailed observations and deductions in this Paper. From the experimental results, a heat balance-sheet has been drawn up for each trial. From the data thus obtained, a series of curves has been plotted, and by interpolation from these curves the more important data corresponding to speeds of 150,200 , and 250 revolutions per minute, and to brake horse-powers of $10,15,20,25$ and 30 , have been deduced for each gas mixture and for each compression. These are given in Tables 1 to 5 (pages 381-385), while the salient features are shown graphically in the curves of Figs. 3 to 6 (pages 370-373).

The main results of the investigation may be summarized as follows :-

Mechanical Efficiency.-The mechanical efficiency of the engine-

(1) Increases with increasing loads.

(2) Diminishes as the ratio $\frac{\text { air }}{\text { gas }}$ increases. (Fig. 4.)

(3) Diminishes as the speed increases. (Fig. 5.)

(4) Is sensibly independent of the compression ratio. (Fig. 6.)

The maximum efficiency attained in these trials, i.e., at full load with the richest $(7: 1)$ mixture, and at the lowest speed (150 (Continued on page 374 .) 
HEAT DISTRIBUTION IN GAS-ENGLNt CYLINDER. MaY 1915.

FIG. 3.

Compression Ratio $=5 \cdot 17$

R.P.M. $=200 \quad$ Air $:$ Gas $=9: 1$

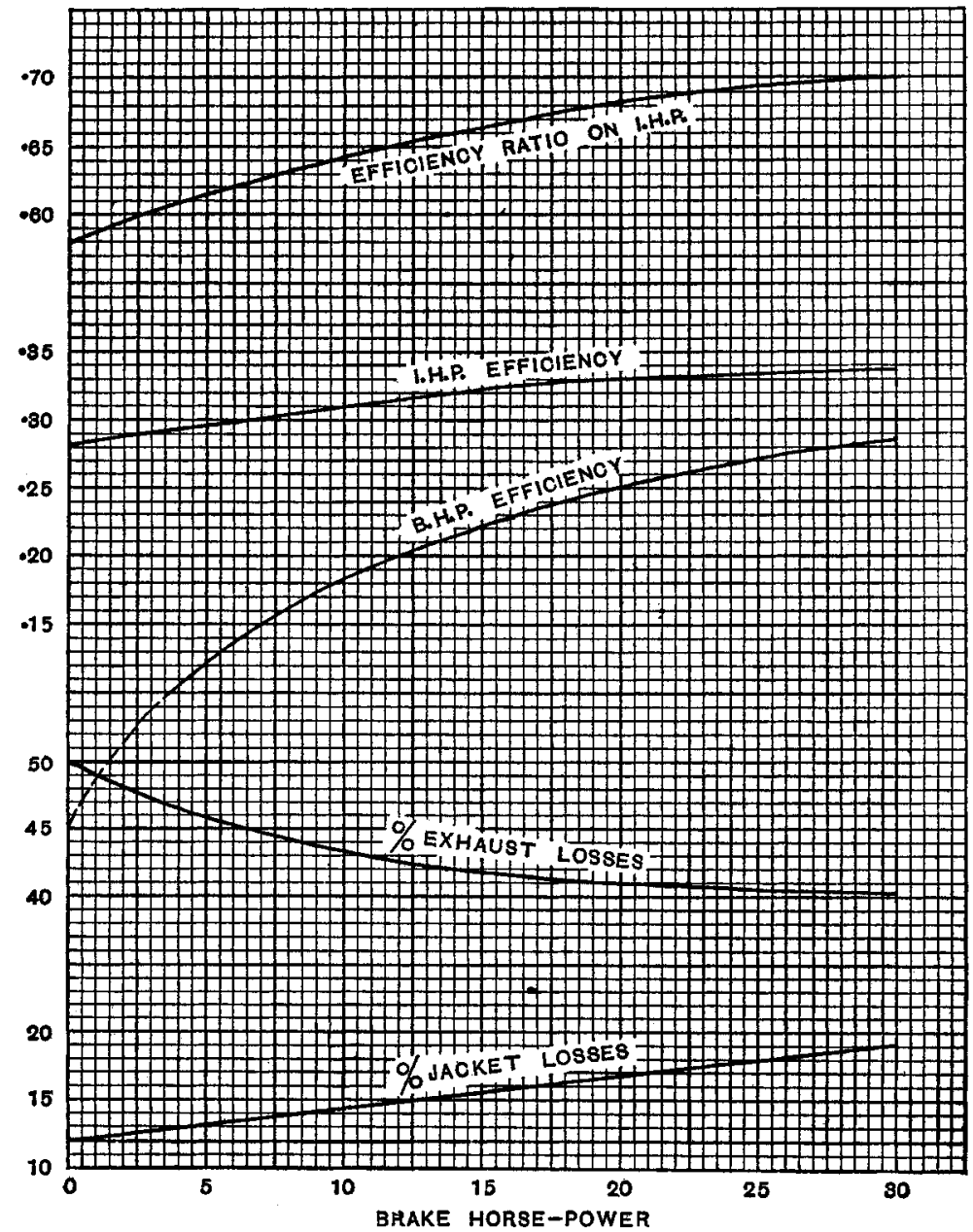

Note.-"Efficiency Ratio" shown at the top of Figs. 3, 4, 5, and 6, would be better substituted by "Relative Efficienoy," according to the suggestion made by Captain Sankey on page 394. 
MAY 1915. HEAT DISTRIBUTION IN GAS-ENGINE CYLINDER.

FIG. 4.

Full Load. $\quad$ Compression Ratio $=5 \cdot 17$

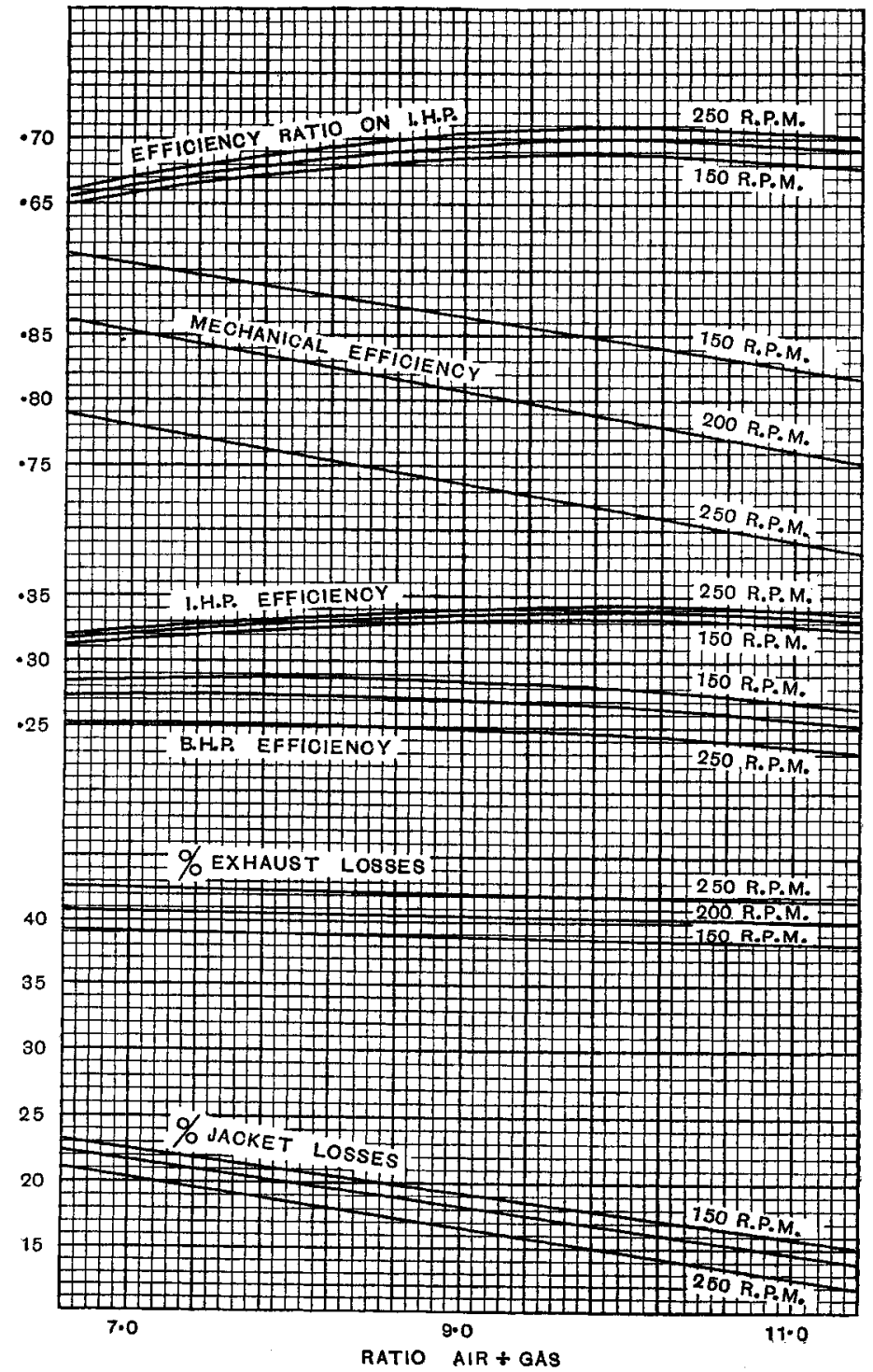


HEAT DISTRIBUTION IN GA8-ENGINE CYLINDER, MAY 1915.

Fig. 5.
Compression Ratio $=5 \cdot 17$
Air $:$ Gas $=9: 1$

Curves AA are from tests at full load.

" BB " , , , 0.8 per cent. full load.

" CC ", , ", , 0.6, , , ,

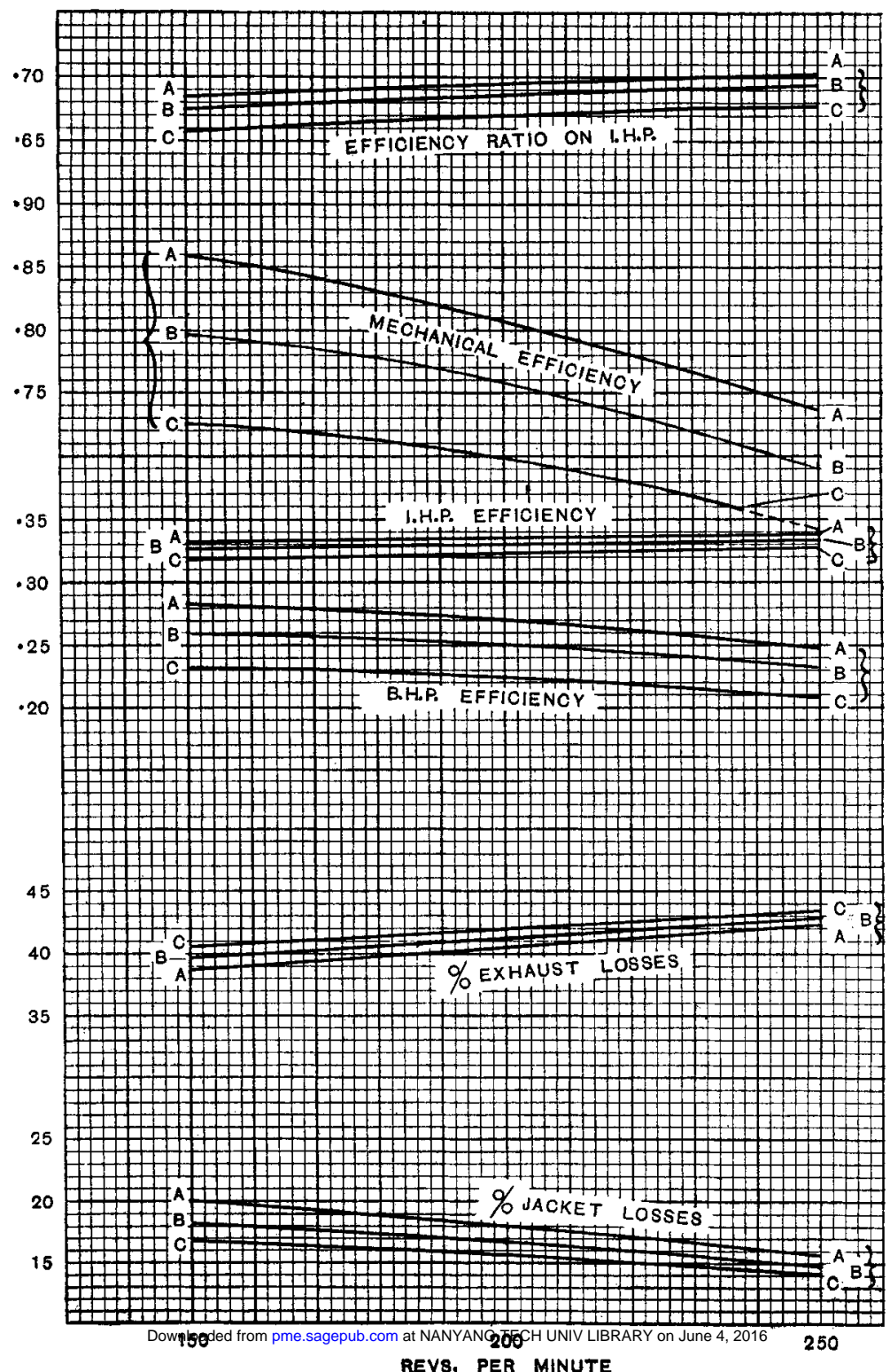


May 1915. HEAT Dis'tribution IN GAS-ENGINE CYLINDER.

Frg. 6.-Full Load. 200 revolutions per minute.

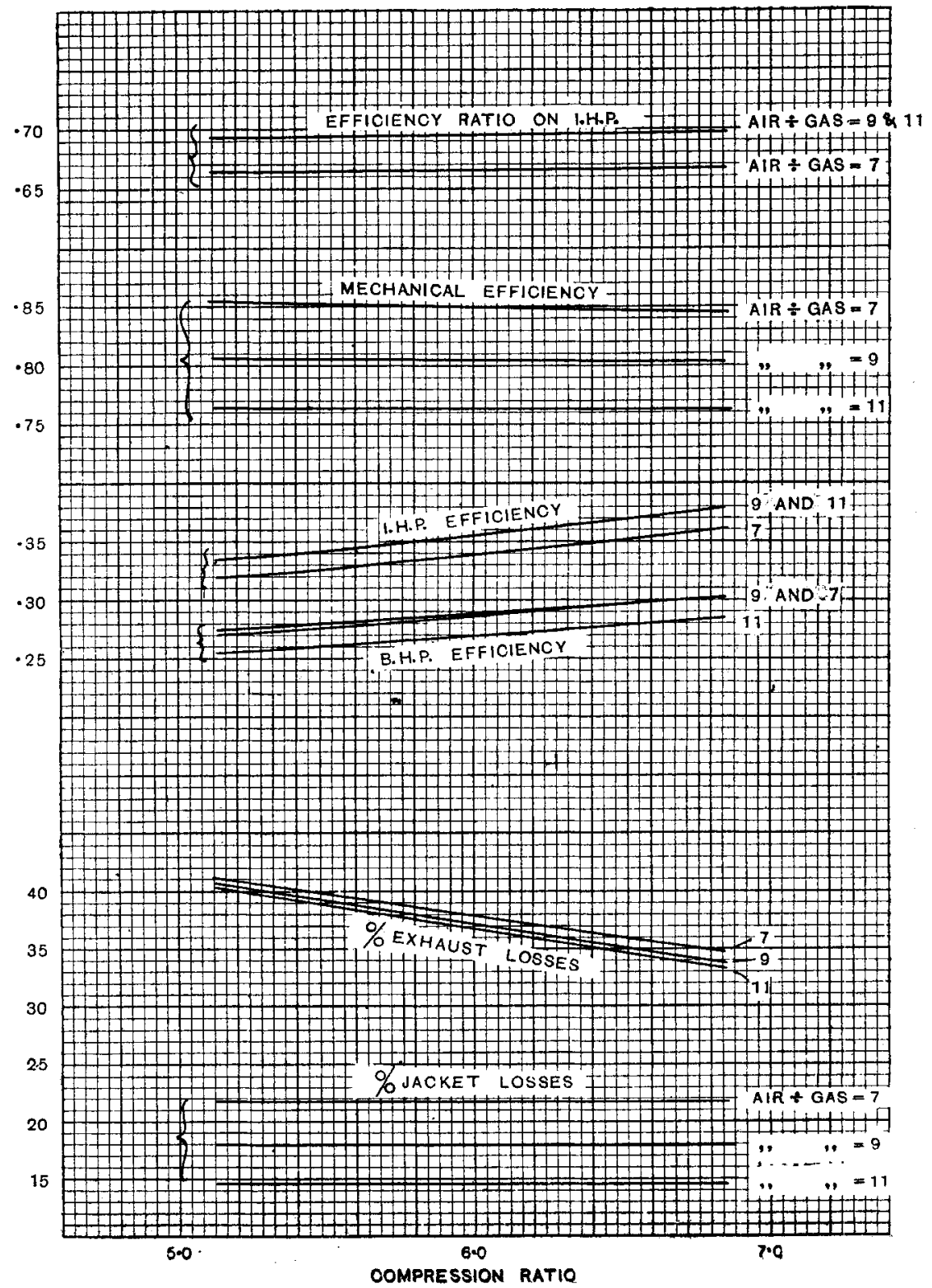


revolutions per minute) is 88 per cent. At the normal speed of 200 revolutions per minute and with the same mixture, the efficiency is 85 per eent., while with this same speed and the weakest $(11: 1)$ mixture, it falls to $76 \cdot 7$ per cent.

Thermal Efficiency on I.H.P.-The thermal efficiency as measured on the i.h.p.-

(1) Increases with the load. (Fig. 3.)

(2) Attains a maximum with an $\frac{\text { air }}{\text { gas }}$ mixture of approximately $10: 1$. (Fig. 4.)

(3) Increases very slightly as the speed increases. (Fig. 5.)

(4) Increases as the compression ratio increases. (Fig. 6.)

The maximum percentage thermal efficiencies attained are as follows :-

\begin{tabular}{|c|c|c|c|c|}
\hline \multicolumn{2}{|c|}{ Compression Ratio. } & $5 \cdot 17$ & $5 \cdot 70$ & $6 \cdot 62$ \\
\hline \multirow{3}{*}{ Speed } & $(150$ & $33 \cdot 1$ & $34 \cdot 4$ & $36 \cdot 5$ \\
\hline & 200 & $33 \cdot 9$ & $35 \cdot 3$ & $37 \cdot 4$ \\
\hline & $(250$ & $34 \cdot 4$ & $35 \cdot 8$ & $37 \cdot 9$ \\
\hline
\end{tabular}

Thermal Efficiency on B.H.P.-As measured on the b.h.p., the thermal efficiency-

(1) Increases with the load. (Fig. 3.)

(2) Attains a maximum with an $\frac{\text { air }}{\text { gas }}$ ratio of $8: 1$, i.e. with a richer mixture than gives maximum i.h.p. efficiency. (Fig. 4.)

(3) Diminishes as speed increases. (Fig. 5.)

(4) Increases with the compression ratio. (Fig. 6.)

The maximum percentage-efficiencies on the b.h.p. are as follows:-

\begin{tabular}{|c|c|c|c|}
\hline Compression Ratio. & $5 \cdot 17$ & $5 \cdot 70$ & $6 \cdot 62$ \\
\hline Speed $\left\{\begin{array}{l}150 \\
200 \\
250\end{array}\right.$ & $\begin{array}{l}27 \cdot 9 \\
27 \cdot 5 \\
25 \cdot 7\end{array}$ & $\begin{array}{l}29 \cdot 1 \\
28 \cdot 6 \\
26 \cdot 7\end{array}$ & $\begin{array}{l}31 \cdot 0 \\
30 \cdot 2 \\
28 \cdot 3\end{array}$ \\
\hline
\end{tabular}


Efficiency Ratio.--Adopting the air cycle as the standard of comparison, the ideal efficiencies corresponding to the various compression ratios are:-

\begin{tabular}{|l|l|l|l|l|}
\hline Compression ratio.. & $5 \cdot 17$ & $5 \cdot 70$ & 6.62 \\
Air-cycle efficiency. & $\cdot$ & 0.482 & 0.501 & 0.532 \\
\hline
\end{tabular}

The ratio of the actual thermal efficiency, measured on the i.h.p., to the corresponding air-cycle efficiency-

(1) Increases with the load. (Fig. 3.)

(2) Has a maximum value when the ratio ${ }_{\text {gas }}^{\text {air }}$ is approximately $10: 1$. (Fig. 4.)

(3) Increases slightly with speed. (Fig. 5.)

(4) Is sensibly independent of the compression ratio. (Fig. 6.)

At full load, and with the most efficient air-gas mixture, the relative efficiencies are, for all compressions :-

\begin{tabular}{|lcc|c|c|c|}
\hline Revolutions & $\cdot$ & $\cdot$ & 150 & 200 & 250 \\
Effficiency ratio & $\cdot$ & $\cdot$ & 0.687 & 0.700 & 0.709 \\
\hline
\end{tabular}

Exhaust-Losses.-The percentage exhaust-losses-

(1) Diminish as the load increases. (Fig. 3.)

(2) Diminish very slightly as the ratio $\frac{\text { air }}{\text { gas }}$ increases. (Fig. 4.)

(3) Increase as the speed increases. (Fig. 5.)

(4) Diminish as the compression ratio increases. (Fig. 6.)

At full load the exhaust-losses in these trials lie between the limits 33.6 per cent. and 42.5 per cent. The former value corresponds to a weak mixture, high compression, and low speed, and the lattier to a rich mixture, low compression ratio, and high speed.

Jacket-Losses. - The percentage heat carried away by the water flowing through the cylinder jackets $\ldots$ not including the exhaust. valve jacket- 
(1) Increases with the load. (Fig. 3.)

(2) Diminishes as the ratio $\frac{\text { air }}{\text { gas }}$ increases. (Fig. 4.)

(3) Diminishes as the speed increases. (Fig. 5.)

(4) Is sensibly independent of the compression-ratio. (Fig. 6.)

With the strongest mixture, and at full load, the percentage heat transmitted to these jackets is $1 \cdot 10$ times as great at 150 revolutions per minute as at 250 revolutions per minute, while with the weakest mixture the ratio becomes $1 \cdot 23$. Since at 150 revolutions per minute the period of contact per cycle, of hot gases and cylinder walls, is 1.66 times as great as at 250 revolutions per minute, the rate of transmission of heat through the cooling surfaces is evidently much greater at the highest speed. An examination of the indicator diagrams, moreover, shows that the maximum pressure and temperature attained in the cylinder is approximately 6 per cent. greater at 150 than at 250 revolutions per minute, so that this increased rate of transmission is obtained in spite of a lower gas temperature. The reason is apparently to be found in the fact that the greater turbulence of the working fluid at the higher rates of speed increases its effective conductivity to an extent which more than counterbalances the effects of a smaller temperature difference and a shortened time of contact. Other things being equal, a 6 per cent. increase in the temperature of the gases would probably increase the heat transmitted by conduction and radiation by some 15 per cent., so that it may be taken approximately that the effective conductivity is increased in the same ratio as the speed of the engine.

Radiation-Losses.-The radiation-loss-that is, the balance of the heat accounted for as indicated work, in the exhaust and in the jacket water-

(1) Diminishes as the load increases. (Table 3, page 383.)

(2) Increases as the ratio $\frac{\text { air }}{\text { gas }}$ increases. (Table 3.)

(3) Diminishes as the speed increases. (Table 3.)

(4) Increases slightly as the compression-ratio increases. (Table 3.) 
At full load, radiation accounts for between 5 per cent. and 14 per cent. of the heat given to the engine, the former value obtaining with a rich mixture, high speed, and low compressionratio, and the latter with a weak mixture, low speed, and high compression-ratio. Its variation with load is indicated in the following Table:-

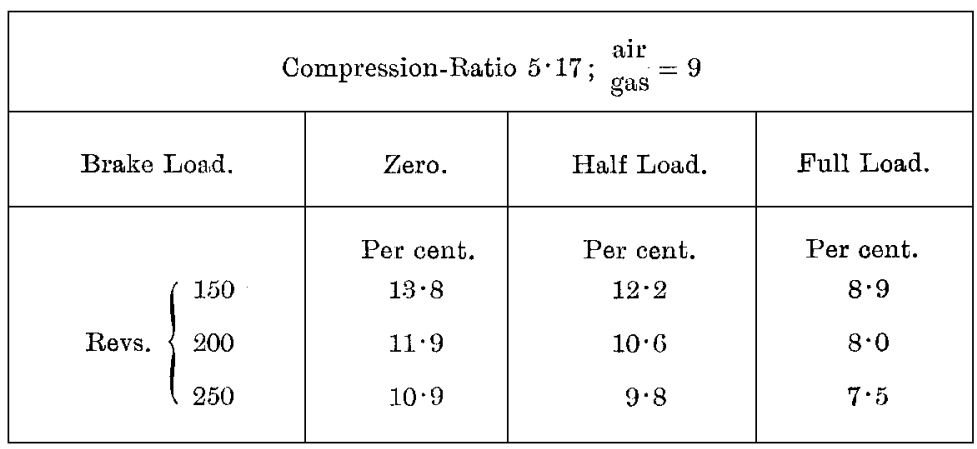

Distribution of Heat up to end of Expansion Stroke. - Since part of the heat carried away by the jacket water passes into the cylinder walls after release, this should, in a true heat-balance, be credited to exhaust. The item attributed to radiation represents heat lost by radiation from the hot exposed surfaces of the piston and of the unjacketed portion of the breech, and from the outer surface of the jackets. Although this loss is wholly due to heat flow through the walls, only part of this flow takes place during the expansion stroke. The remainder, occurring after the end of this stroke, is also to be attributed to the exhaust.

Thus in a heat-balance drawn for the working fluid up to the end of expansion, the apparent heat flow into the walls is to be increased by the greater part of this radiation-loss, and to be diminished by that part of the heat transmitted to the jacket water during exhaust. Similarly the apparent exhaust-losses are to be increased by some small portion of the radiation-loss and by that part of the heat given to the jacket water during exhaust. The net result is that both the wall-losses and the exhaust-losses as given

2 c 2 
by direct measurement are to be increased by some unknown proportion of the radiation-loss.

A true heat-balance for this portion of the cycle can only be deduced if the heat given per cycle in the combustible gas, the work done per cycle, and the energy of the working fluid at the end of expansion, are known. In order to determine the energy at the end of expansion, it is necessary to know :-

(1) The temperature at the end of expansion.

(2) The quantity of the working fluid in the cylinder.

(3) Its internal energy per cubic foot in terms of its temperature.

In these trials all the data necessary for determining items (1) and (2) are available. The temperatures at the end of expansion have been deduced from a knowledge of the temperatures at the beginning of compression and of the pressures at the end of expansion and at the beginning of compression, while the temperatures at the beginning of compression have been calculated by the method outlined by Dugald Clerk.* The internal energy per cubic foot corresponding to the various release temperatures has been deduced from curves given by Hopkinson. $\dagger$ The mean results of these calculations, carried out for a number of the trials, are embodied in the heat-balances given in Table 5 (page 385). Since their accuracy depends on that of the indicator diagrams, the possibility of error in these should not be overlooked. It is unlikely, however, that the error in any item of these balancesheets exceeds about 1 per cent., while it may be much less.

The results indicate in general, that of the total radiation-loss obtained by difference from the heat measurements, a proportion ranging from about 0.33 to 0.40 is to be added to the apparent exhaust-losses, the remainder going to increase the apparent jacket or wall losses. This proportion attains its highest value with the highest compression-ratios and with the richest mixtures.

A comparison of these with results obtained in a similar

* Proceedings, Inst. C.E., 1907, vol. clxix, page 148.

$\dagger$ Proceedings, I.Mech.E., 1908, page 424. 
manner by Hopkinson* on a slightly larger engine shows a fairly close agreement. In round numbers the figures are as follows. Those attributed to the authors are obtained by interpolation from the figures of Table 5 .

\begin{tabular}{|c|c|c|c|c|}
\hline \multirow{2}{*}{-} & \multicolumn{2}{|c|}{$\frac{\text { Air }}{\text { Gas }}=10.8$} & \multicolumn{2}{|c|}{$\frac{\text { Air }}{\overline{G a S}}=8 \cdot 1$} \\
\hline & Hopkinson. & Authors. & Hopkinson. & Authors. \\
\hline Heat as i.h.p. & 37 & 36 & 33 & 35 \\
\hline Heat in exhaust & 42 & 41 & 39 & 39 \\
\hline Heat flow to walls. & 21 & 23 & 28 & 26 \\
\hline
\end{tabular}

The engine used by Hopkinson had a cylinder diameter $=1.1 \cdot 5$ inches ; stroke $=21$ inches; compression-ratio $=6 \cdot 37$; revolutions $=110$ per minute.

Heat entering Exhanst-valve Jacket. - The percentage heat entering the exhaust-valve jacket is shown in Table 3 (page 383). From these figures it appears that its value ranges from 8.0 to 12.5 per cent., being greatest at low loads, low speeds, and with low compression-ratios and rich mixtures. At full load it varies as shown in the following Table :-

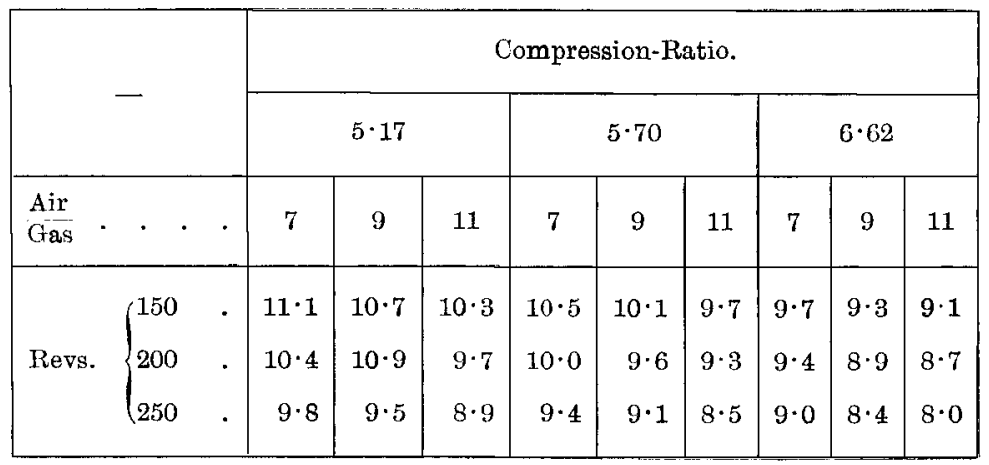

* Proceedings, I.Mech.E., 1908, page 417. 
If this be added to the jacket-loss given in Table 4 (page 384), it gives the jacket-loss as determined from trials of an engine fitted with the usual arrangement of jackets. It is interesting to note in this connexion, that with weak mixtures and fairly high compression-ratios, the jacket-losses obtained in this way are in substantial agreement with those obtained by analysis as giving the heat flow during expansion. For example, at full load, with $\frac{\text { air }}{\text { gas }}$ mixture $11: 1$ and with compression-ratio $6 \cdot 62$, the percentage wall-losses by analysis at speeds of 150,200 , and 250 revolutions per minute are respectively $24 \cdot 6,23 \cdot 3$, and $21 \cdot 3$, Table 5 (page 385 ), while the percentages of heat to barrel and valve jackets are respectively $24 \cdot 9,23 \cdot 5$, and $20 \cdot 9$. Under favourable circumstances it appears that a heat balance-sheet obtained by measuring the indicated work and jacket heat of a commercial type of engine, and by estimating exhaust-losses by difference, is in extremely close agreement with the balance-sheet based on the internal energy of the gas at the end of expansion. For fairly rich mixtures and lower compression-ratios the measured jacket-losses are, however, always in excess of those more correctly computed from the internal energy of the gas.

The authors would express their sense of indebtedness to the Trustees of the Carnegie Research Fund, whose scholarship to Mr. Walker rendered possible his co-operation in the work. Also to Mr. Linton, of the Engineering Staff of University College, for much assistance given during the trials.

The Paper is illustrated by 6 Figs. in the letterpress. 
MAY 1915. HEAT DISTRIBUTION IN GAS-ENGINE CYLINDER.

TABLE 1.

Thermal Efficiencies : (a) on I.H.P., and $(b)$ on B.H.P.

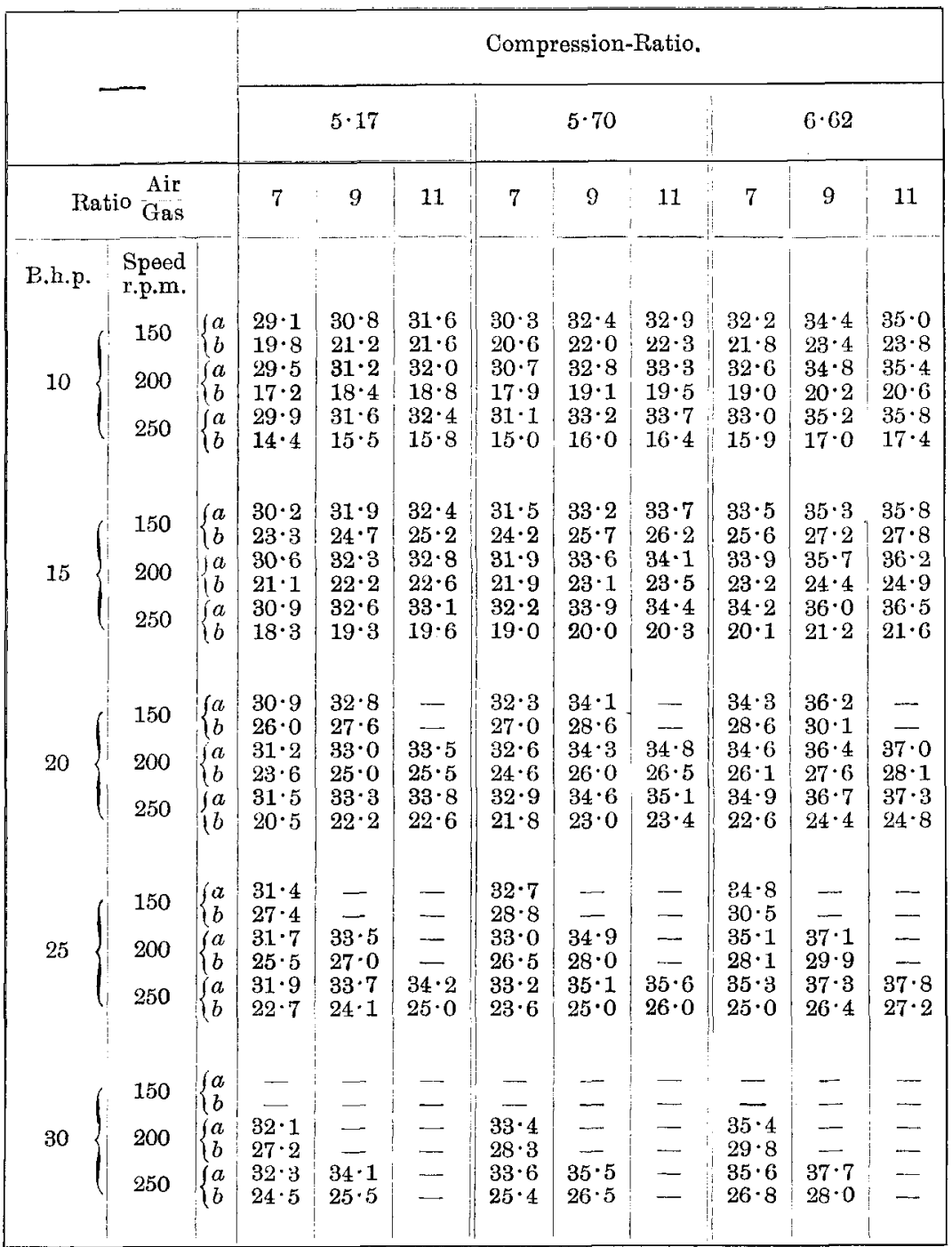


TABLE 2.-(a) Heat in Exhaust; (b) Heat transferred to Barrel Jacket-water; expressed as a percentage of the total Heat Supply.

Jacket-losses are sensibly independent of the compression-ratio.

\begin{tabular}{|c|c|c|c|c|c|c|c|c|c|c|c|}
\hline \multirow{3}{*}{\multicolumn{3}{|c|}{$\frac{-}{\text { Ratio } \frac{\text { Air }}{\text { Gas }}}$}} & \multicolumn{9}{|c|}{ Compression-Ratio. } \\
\hline & & & \multicolumn{3}{|c|}{$5 \cdot 17$} & \multicolumn{3}{|c|}{$5 \cdot 70$} & \multicolumn{3}{|c|}{$6 \cdot 62$} \\
\hline & & & 7 & 9 & 11 & 7 & 9 & 11 & 7 & 9 & 11 \\
\hline B.h.p. & $\begin{array}{l}\text { Speed } \\
\text { r.p.m. }\end{array}$ & & & & & & & & & & \\
\hline 10 & $\begin{array}{l}150 \\
200 \\
250\end{array}$ & $\left\{\begin{array}{l}\left\{\begin{array}{l}a \\
b \\
a \\
b \\
a \\
b\end{array}\right. \\
0\end{array}\right.$ & $\begin{array}{l}42 \cdot 1 \\
17 \cdot 0 \\
44 \cdot 1 \\
16 \cdot 0 \\
46 \cdot 1 \\
15 \cdot 0\end{array}$ & $\begin{array}{l}41 \cdot 0 \\
16 \cdot 0 \\
43 \cdot 2 \\
14 \cdot 3 \\
45 \cdot 4 \\
12 \cdot 6\end{array}$ & $\begin{array}{l}40 \cdot 3 \\
14 \cdot \cdot \\
42 \cdot 3 \\
12 \cdot 2 \\
44 \cdot 4 \\
10 \cdot 3\end{array}$ & $\begin{array}{l}39 \cdot 9 \\
17 \cdot 4 \\
41 \cdot 4 \\
16 \cdot 3 \\
43 \cdot 4 \\
15 \cdot 3\end{array}$ & $\begin{array}{l}38 \cdot 4 \\
16 \cdot 0 \\
40 \cdot 7 \\
14 \cdot 3 \\
42 \cdot 7 \\
12 \cdot 6\end{array}$ & $\begin{array}{l}37 \cdot 8 \\
14 \cdot 1 \\
39 \cdot 8 \\
12 \cdot 2 \\
41 \cdot 9 \\
10 \cdot 3\end{array}$ & $\begin{array}{l}36 \cdot 2 \\
17 \cdot 0 \\
38 \cdot 1 \\
16 \cdot 3 \\
40 \cdot 0 \\
15 \cdot 3\end{array}$ & $\begin{array}{l}35 \cdot 2 \\
16 \cdot 0 \\
37 \cdot 3 \\
14 \cdot 3 \\
39 \cdot 3 \\
12 \cdot 6\end{array}$ & $\begin{array}{l}34 \cdot 3 \\
14 \cdot 1 \\
36 \cdot 4 \\
12 \cdot 2 \\
38 \cdot 5 \\
10 \cdot 3\end{array}$ \\
\hline & 1.50 & $\left\{\begin{array}{l}a \\
b\end{array}\right.$ & $\begin{array}{l}40 \cdot 8 \\
18.6\end{array}$ & $\begin{array}{l}39 \cdot 7 \\
17 \cdot 6\end{array}$ & $\begin{array}{l}39 \cdot 1 \\
15 \cdot 5\end{array}$ & $38 \cdot 5$ & $\begin{array}{l}37 \cdot 7 \\
-\end{array}$ & $36 \cdot 8$ & $35 \cdot 2$ & $34 \cdot 8$ & $33 \cdot 8$ \\
\hline 15 & 200 & $\left\{\begin{array}{l}a \\
b\end{array}\right.$ & $\begin{array}{l}42 \cdot 7 \\
17 \cdot 4\end{array}$ & $\begin{array}{l}41 \cdot 8 \\
15 \cdot 6\end{array}$ & $\begin{array}{l}41 \cdot 0 \\
13 \cdot 4\end{array}$ & $40 \cdot 3$ & $39 \cdot 6$ & $38 \cdot 7$ & $36 \cdot 9$ & 36.3 & $35 \cdot 5$ \\
\hline & 250 & $\left\{\begin{array}{l}a \\
b\end{array}\right.$ & $\begin{array}{l}44 \cdot 6 \\
16 \cdot 1\end{array}$ & $\begin{array}{l}43 \cdot 9 \\
13 \cdot 7\end{array}$ & $\begin{array}{l}48 \cdot 1 \\
11 \cdot 3\end{array}$ & 42.2 & $41 \cdot 5$ & 40.7 & $\begin{array}{c}38 \cdot 6 \\
-\end{array}$ & $\begin{array}{c}37 \cdot 9 \\
-\end{array}$ & $37 \cdot 1$ \\
\hline & 150 & $\left\{\begin{array}{l}a \\
b\end{array}\right.$ & $\begin{array}{l}39 \cdot 9 \\
20 \cdot 3\end{array}$ & $\begin{array}{l}39 \cdot 1 \\
19 \cdot 2\end{array}$ & z & $37 \cdot 8$ & $37 \cdot 0$ & 二 & $34 \cdot 5$ & $33 \cdot 7$ & - \\
\hline 20 & 200 & $\left\{\begin{array}{l}a \\
b\end{array}\right.$ & $\begin{array}{l}41 \cdot 8 \\
18 \cdot 8\end{array}$ & $\begin{array}{l}41 \cdot 1 \\
16 \cdot 8\end{array}$ & $\begin{array}{l}40 \cdot 1 \\
14 \cdot 6\end{array}$ & $39 \cdot 5$ & $38 \cdot 8$ & $37 \cdot 9$ & $36 \cdot 0$ & $35 \cdot 3$ & $34 \cdot 3$ \\
\hline & 250 & $\left\{\begin{array}{l}a \\
b\end{array}\right.$ & $\begin{array}{l}43 \cdot 7 \\
17 \cdot 2\end{array}$ & $\begin{array}{l}43 \cdot 0 \\
14 \cdot 5\end{array}$ & $\begin{array}{l}42 \cdot 2 \\
12 \cdot 3\end{array}$ & $41 \cdot 3$ & $\begin{array}{c}40 \cdot 6 \\
-\end{array}$ & $\begin{array}{c}39 \cdot 8 \\
-\end{array}$ & $\begin{array}{l}37 \cdot 5 \\
-\end{array}$ & $\begin{array}{c}36 \cdot 8 \\
-\end{array}$ & $36 \cdot 0$ \\
\hline & 150 & $\left\{\begin{array}{l}a \\
b\end{array}\right.$ & $\begin{array}{l}39 \cdot 3 \\
22 \cdot 2\end{array}$ & 二 & 二 & $37 \cdot 3$ & - & - & $34 \cdot 2$ & - & - \\
\hline 25 & 200 & $\left\{\begin{array}{l}a \\
b\end{array}\right.$ & $\begin{array}{l}41 \cdot 2 \cdot 2 \\
20 \cdot 2\end{array}$ & $\begin{array}{l}40 \cdot 5 \\
18 \cdot 0\end{array}$ & 二 & $39 \cdot 2$ & $38 \cdot 4$ & - & $35 \cdot 6$ & $34 \cdot 9$ & - \\
\hline & 250 & $\left\{\begin{array}{l}a \\
b\end{array}\right.$ & $\begin{array}{l}43 \cdot 1 \\
18 \cdot 3\end{array}$ & $\begin{array}{l}42 \cdot 4 \\
15 \cdot 3\end{array}$ & $\begin{array}{l}41 \cdot 6 \\
13 \cdot 3\end{array}$ & $41 \cdot 0$ & $\begin{array}{c}40 \cdot 3 \\
-\end{array}$ & $39 \cdot 5$ & $37 \cdot 0$ & $36 \cdot 3$ & $35 \cdot 5$ \\
\hline & 150 & $\left\{\begin{array}{l}a \\
b\end{array}\right.$ & - & - & - & - & - & - & - & - & - \\
\hline 30 & 200 & $\left\{\begin{array}{l}a \\
b\end{array}\right.$ & $40 \cdot 8$ & - & - & $39 \cdot 0$ & - & - & $35 \cdot 4$ & - & - \\
\hline & 250 & $\left\{\begin{array}{l}a \\
b\end{array}\right.$ & $\begin{array}{l}42 \cdot 7 \\
19 \cdot 5\end{array}$ & $\begin{array}{l}41 \cdot 9 \\
16 \cdot 1\end{array}$ & $=$ & $40 \cdot 8$ & $40 \cdot 1$ & - & $36 \cdot 8$ & $36 \cdot 1$ & $\overline{-}$ \\
\hline
\end{tabular}


MAY 1915. HEAT DISTRIBUTION IN GAS-ENGINE CYLINDER.

TABLE 3.

Radiation-Loss (by difference) ; (b) Heat to Exhaust-valve jacket; expressed as a percentage of the total Heat Supply.

\begin{tabular}{|c|c|c|c|c|c|c|c|c|c|c|c|}
\hline & & & \multicolumn{9}{|c|}{ Compression-Ratio. } \\
\hline & & & & $5 \cdot 17$ & & & $5 \cdot 70$ & & & $6 \cdot 62$ & \\
\hline \multicolumn{3}{|c|}{ Ratio $\frac{\text { Air }}{\text { Gas }}$} & 7 & 9 & 11 & 7 & 9 & 11 & 7 & 9 & 11 \\
\hline B.h.p. & $\begin{array}{l}\text { Speed } \\
\text { r.p.m. }\end{array}$ & & & & & & & & & & \\
\hline 10 & $\begin{array}{l}150 \\
200 \\
250\end{array}$ & $\left\{\begin{array}{l}\left\{\begin{array}{l}a \\
b\end{array}\right. \\
\left\{\begin{array}{l}a \\
b\end{array}\right. \\
\left\{\begin{array}{l}a \\
b\end{array}\right.\end{array}\right.$ & $\begin{array}{r}11.8 \\
12.5 \\
10.4 \\
11.2 \\
9.0 \\
10.4\end{array}$ & $\begin{array}{r}12 \cdot 2 \\
11 \cdot 4 \\
11 \cdot 3 \\
10 \cdot 4 \\
10 \cdot 4 \\
9 \cdot 8\end{array}$ & $\begin{array}{r}14 \cdot 0 \\
10 \cdot 8 \\
13 \cdot 5 \\
9 \cdot 9 \\
12 \cdot 9 \\
9 \cdot 1\end{array}$ & $\begin{array}{l}12 \cdot 8 \\
11.5 \\
11.6 \\
10 \cdot 6 \\
10 \cdot 2 \\
10.0\end{array}$ & $\begin{array}{r}13 \cdot 2 \\
10 \cdot 8 \\
12 \cdot 2 \\
10 \cdot 0 \\
11 \cdot 5 \\
9 \cdot 5\end{array}$ & $\begin{array}{r}15 \cdot 2 \\
10 \cdot 4 \\
14 \cdot 7 \\
9 \cdot 6 \\
14 \cdot 1 \\
8 \cdot 9\end{array}$ & $\begin{array}{r}14 \cdot 2 \\
10 \cdot 2 \\
18 \cdot 3 \\
9 \cdot 8 \\
12 \cdot 0 \\
9 \cdot 5\end{array}$ & $\begin{array}{r}14 \cdot 4 \\
10 \cdot 0 \\
13 \cdot 6 \\
9 \cdot 4 \\
12 \cdot 9 \\
8 \cdot 9\end{array}$ & $\begin{array}{r}16 \cdot 2 \\
9 \cdot 9 \\
16 \cdot 0 \\
9 \cdot 2 \\
15 \cdot 4 \\
8 \cdot 6\end{array}$ \\
\hline 15 & $\begin{array}{l}150 \\
200 \\
250\end{array}$ & $\left\{\begin{array}{l}a \\
b \\
a \\
b \\
a \\
b\end{array}\right.$ & $\begin{array}{r}10 \cdot 4 \\
11 \cdot 8 \\
9.3 \\
10 \cdot 8 \\
8.4 \\
10 \cdot 2\end{array}$ & $\begin{array}{r}10 \cdot 8 \\
11 \cdot 0 \\
10 \cdot 3 \\
10 \cdot 2 \\
9 \cdot 8 \\
9 \cdot 7\end{array}$ & $\begin{array}{r}13 \cdot 0 \\
10 \cdot 5 \\
12 \cdot 8 \\
9 \cdot 8 \\
12 \cdot 5 \\
9.0\end{array}$ & $\begin{array}{r}11 \cdot 4 \\
11 \cdot 0 \\
10 \cdot 4 \\
10 \cdot 3 \\
9 \cdot 5 \\
9 \cdot 8\end{array}$ & $\begin{array}{r}11 \cdot 7 \\
10 \cdot 4 \\
11 \cdot 2 \\
9 \cdot 8 \\
10 \cdot 9 \\
9 \cdot 3\end{array}$ & $\begin{array}{r}14 \cdot 0 \\
10 \cdot 0 \\
13 \cdot 8 \\
9 \cdot 4 \\
13 \cdot 6 \\
8 \cdot 7\end{array}$ & $\begin{array}{r}12 \cdot 7 \\
10 \cdot 0 \\
11 \cdot 8 \\
9 \cdot 6 \\
11 \cdot 1 \\
9 \cdot 2\end{array}$ & $\begin{array}{r}12 \cdot 3 \\
9 \cdot 6 \\
12 \cdot 4 \\
9 \cdot 1 \\
12 \cdot 4 \\
8 \cdot 6\end{array}$ & $\begin{array}{r}14 \cdot 8 \\
9 \cdot 4 \\
14 \cdot 9 \\
8 \cdot 8 \\
15 \cdot 1 \\
8 \cdot 2\end{array}$ \\
\hline 20 & $\begin{array}{l}150 \\
200 \\
250\end{array}$ & $\left\{\begin{array}{l}a \\
b \\
a \\
b \\
a \\
b\end{array}\right.$ & $\begin{array}{r}8.9 \\
11.4 \\
8.2 \\
10.5 \\
7.6 \\
10.0\end{array}$ & $\begin{array}{r}9 \cdot 0 \\
10 \cdot 7 \\
9 \cdot 1 \\
10 \cdot 0 \\
9 \cdot 2 \\
9 \cdot 6\end{array}$ & $\begin{array}{r}- \\
11.8 \\
9.7 \\
11.8 \\
8.9\end{array}$ & $\begin{array}{r}9 \cdot 6 \\
10 \cdot 7 \\
9 \cdot 1 \\
10 \cdot 1 \\
8 \cdot 6 \\
9 \cdot 6\end{array}$ & $\begin{array}{r}9 \cdot 8 \\
10 \cdot 1 \\
10 \cdot 1 \\
9 \cdot 7 \\
10 \cdot 3 \\
9 \cdot 2\end{array}$ & $\begin{array}{r}- \\
12 \cdot 7 \\
9 \cdot 3 \\
12 \cdot 8 \\
8 \cdot 5\end{array}$ & $\begin{array}{r}10.9 \\
9.9 \\
10.6 \\
9.5 \\
10 \cdot 4 \\
9.1\end{array}$ & $\begin{array}{r}11 \cdot 0 \\
9 \cdot 3 \\
11 \cdot 5 \\
9 \cdot 0 \\
12 \cdot 0 \\
8 \cdot 5\end{array}$ & $\begin{array}{r}- \\
- \\
14 \cdot 0 \\
8 \cdot 7 \\
14 \cdot 4 \\
8 \cdot 0\end{array}$ \\
\hline 25 & $\begin{array}{l}150 \\
200 \\
250\end{array}$ & $\left\{\begin{array}{l}a \\
b \\
a \\
a b \\
\left\{\begin{array}{l}a \\
b\end{array}\right.\end{array}\right.$ & $\begin{array}{r}7 \cdot 1 \\
11 \cdot 2 \\
6 \cdot 9 \\
10 \cdot 4 \\
6 \cdot 7 \\
10 \cdot 0\end{array}$ & $\begin{array}{l}- \\
8 \cdot 0 \\
9 \cdot 9 \\
8 \cdot 6 \\
9 \cdot 6\end{array}$ & $\begin{array}{l}- \\
- \\
\overline{9} \\
8 \cdot 9\end{array}$ & $\begin{array}{r}7 \cdot 8 \\
10 \cdot 6 \\
7 \cdot 6 \\
10 \cdot 0 \\
7 \cdot 5 \\
9 \cdot 6\end{array}$ & $\begin{array}{l}- \\
8 \cdot 7 \\
9 \cdot 6 \\
9 \cdot 3 \\
9 \cdot 1\end{array}$ & $\begin{array}{l}- \\
= \\
\overline{11 \cdot 6} \\
8 \cdot 5\end{array}$ & $\begin{array}{l}8 \cdot 8 \\
9 \cdot 8 \\
9 \cdot 1 \\
9 \cdot 4 \\
9 \cdot 4 \\
9 \cdot 0\end{array}$ & $\begin{array}{r}- \\
\overline{10} \cdot 0 \\
8 \cdot 9 \\
11 \cdot 1 \\
8 \cdot 3\end{array}$ & $\begin{array}{r}- \\
\overline{-} \\
13 \cdot 4 \\
7 \cdot 9\end{array}$ \\
\hline 30 & $\begin{array}{l}150 \\
200 \\
250\end{array}$ & $\left\{\begin{array}{l}a \\
b \\
a \\
b \\
a \\
b\end{array}\right.$ & $\begin{array}{r}- \\
\overline{5} \cdot 5 \\
10 \cdot 4 \\
5 \cdot 5 \\
9 \cdot 9\end{array}$ & $\begin{array}{l}- \\
\overline{-} \\
\overline{7} \cdot 9 \\
9 \cdot 5\end{array}$ & $\begin{array}{l}- \\
- \\
- \\
-\end{array}$ & $\begin{array}{r}- \\
\overline{6} \cdot 0 \\
10 \cdot 0 \\
6 \cdot 1 \\
9 \cdot 5\end{array}$ & $\begin{array}{l}- \\
\overline{-} \\
\overline{8 \cdot 3} \\
9 \cdot 0\end{array}$ & $\begin{array}{l}- \\
- \\
- \\
-\end{array}$ & $\begin{array}{l}- \\
\overline{7} \cdot 6 \\
9 \cdot 4 \\
8 \cdot 1 \\
9 \cdot 0\end{array}$ & $\begin{array}{c}- \\
\overline{-} \\
\overline{10 \cdot 1} \\
8 \cdot 2\end{array}$ & $\begin{array}{l}E \\
E \\
-\end{array}$ \\
\hline
\end{tabular}


TABLE 4.

Heat Balance at Full Load-i.e., with one Explosion per Cycle.

\begin{tabular}{|c|c|c|c|c|c|c|c|c|c|}
\hline \multirow[b]{2}{*}{$\frac{\text { Air }}{\text { Gas }}$} & \multirow[b]{2}{*}{$\begin{array}{l}\text { Compres- } \\
\text { sion-Ratio. }\end{array}$} & \multirow[b]{2}{*}{$\begin{array}{l}\text { Speed } \\
\text { r.p.m. }\end{array}$} & \multicolumn{4}{|c|}{$\begin{array}{l}\text { Percentage of Heat } \\
\text { Distribution. }\end{array}$} & \multicolumn{2}{|c|}{$\begin{array}{l}\text { Efficiency } \\
\text { Ratio on }\end{array}$} & \multirow[b]{2}{*}{$\begin{array}{l}\text { Heat as } \\
\text { b.h.p. }\end{array}$} \\
\hline & & & 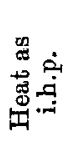 & 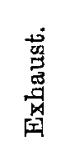 & 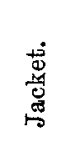 & 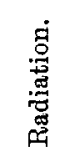 & $\stackrel{\dot{q}}{\dot{q}}$ & 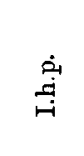 & \\
\hline & $5 \cdot 17$ & $\begin{array}{l}150 \\
200 \\
250\end{array}$ & $\begin{array}{l}31 \cdot 5 \\
32 \cdot 2 \\
32 \cdot 6\end{array}$ & $\begin{array}{l}39 \cdot 2 \\
40 \cdot 7 \\
42 \cdot 5\end{array}$ & $\begin{array}{l}22 \cdot 4 \\
21 \cdot 7 \\
20 \cdot 2\end{array}$ & $\begin{array}{l}6 \cdot 9 \\
5 \cdot 4 \\
4 \cdot 7\end{array}$ & $\begin{array}{l}0.575 \\
0.569 \\
0.531\end{array}$ & $\begin{array}{l}0.654 \\
0.668 \\
0.676\end{array}$ & $\begin{array}{l}27 \cdot 7 \\
27 \cdot 4 \\
25 \cdot 6\end{array}$ \\
\hline 7 & $5 \cdot 70$ & $\begin{array}{l}150 \\
200 \\
250\end{array}$ & $\begin{array}{l}32 \cdot 8 \\
33 \cdot 5 \\
33 \cdot 8\end{array}$ & $\begin{array}{l}37 \cdot 2 \\
38 \cdot 9 \\
40 \cdot 6\end{array}$ & $\begin{array}{l}22 \cdot 4 \\
21 \cdot 7 \\
20 \cdot 2\end{array}$ & $\begin{array}{l}7 \cdot 6 \\
5 \cdot 9 \\
5 \cdot 4\end{array}$ & $\begin{array}{l}0.578 \\
0.568 \\
0.529\end{array}$ & $\begin{array}{l}0.654 \\
0.667 \\
0.674\end{array}$ & $\begin{array}{l}29 \cdot 0 \\
28 \cdot 5 \\
26 \cdot 5\end{array}$ \\
\hline & $6 \cdot 62$ & $\begin{array}{l}150 \\
200 \\
250\end{array}$ & $\begin{array}{l}34 \cdot 9 \\
35 \cdot 5 \\
35 \cdot 8\end{array}$ & $\begin{array}{l}34 \cdot 1 \\
35 \cdot 3 \\
36 \cdot 7\end{array}$ & $\begin{array}{l}22 \cdot 4 \\
21 \cdot 7 \\
20 \cdot 2\end{array}$ & $\begin{array}{l}8 \cdot 6 \\
7 \cdot 5 \\
7 \cdot 3\end{array}$ & $\begin{array}{l}0.579 \\
0.565 \\
0.530\end{array}$ & $\begin{array}{l}0.656 \\
0.668 \\
0.673\end{array}$ & $\begin{array}{l}30 \cdot 8 \\
30 \cdot 0 \\
28 \cdot 2\end{array}$ \\
\hline & $5 \cdot 17$ & $\begin{array}{l}150 \\
200 \\
250\end{array}$ & $\begin{array}{l}32 \cdot 8 \\
33 \cdot 5 \\
34 \cdot 0\end{array}$ & $\begin{array}{l}39 \cdot 1 \\
40 \cdot 5 \\
42 \cdot 0\end{array}$ & $\begin{array}{l}19 \cdot 2 \\
18 \cdot 0 \\
16 \cdot 5\end{array}$ & $\begin{array}{l}8 \cdot 9 \\
8 \cdot 0 \\
7 \cdot 5\end{array}$ & $\begin{array}{l}0.572 \\
0.560 \\
0.521\end{array}$ & $\begin{array}{l}0.680 \\
0.695 \\
0.705\end{array}$ & $\begin{array}{l}27 \cdot 6 \\
27 \cdot 0 \\
25 \cdot 1\end{array}$ \\
\hline 9 & $5 \cdot 70$ & $\begin{array}{l}150 \\
200 \\
250\end{array}$ & $\begin{array}{l}34 \cdot 1 \\
34 \cdot 9 \\
35 \cdot 4\end{array}$ & $\begin{array}{l}37 \cdot 0 \\
38 \cdot 4 \\
40 \cdot 3\end{array}$ & $\begin{array}{l}19 \cdot 2 \\
18 \cdot 0 \\
16 \cdot 5\end{array}$ & $\begin{array}{l}9 \cdot 7 \\
8 \cdot 7 \\
7 \cdot 8\end{array}$ & $\begin{array}{l}0.570 \\
0.560 \\
0.522\end{array}$ & $\begin{array}{l}0.679 \\
0.695 \\
0.705\end{array}$ & $\begin{array}{l}28 \cdot 6 \\
28 \cdot 1 \\
26 \cdot 2\end{array}$ \\
\hline & $6 \cdot 62$ & $\begin{array}{l}150 \\
200 \\
250\end{array}$ & $\begin{array}{l}36 \cdot 2 \\
37 \cdot 1 \\
37 \cdot 6\end{array}$ & $\begin{array}{l}33 \cdot 7 \\
34 \cdot 9 \\
36 \cdot 3\end{array}$ & $\begin{array}{l}19 \cdot 2 \\
18 \cdot 0 \\
16 \cdot 5\end{array}$ & $\begin{array}{r}10 \cdot 9 \\
10 \cdot 0 \\
9 \cdot 6\end{array}$ & $\begin{array}{l}0.568 \\
0.561 \\
0.520\end{array}$ & $\begin{array}{l}0.681 \\
0.697 \\
0.707\end{array}$ & $\begin{array}{l}30 \cdot 2 \\
29 \cdot 8 \\
27 \cdot 6\end{array}$ \\
\hline & $5 \cdot 17$ & $\begin{array}{l}150 \\
200 \\
250\end{array}$ & $\begin{array}{l}32 \cdot 8 \\
33 \cdot 5 \\
33 \cdot 9\end{array}$ & $\begin{array}{l}38 \cdot 9 \\
40 \cdot 0 \\
41 \cdot 8\end{array}$ & $\begin{array}{l}15 \cdot 8 \\
14 \cdot 8 \\
12 \cdot 9\end{array}$ & $\begin{array}{l}12 \cdot 5 \\
11 \cdot 7 \\
11 \cdot 4\end{array}$ & $\begin{array}{l}0.540 \\
0.534 \\
0.502\end{array}$ & $\begin{array}{l}0.680 \\
0.695 \\
0.704\end{array}$ & $\begin{array}{l}26 \cdot 0 \\
25 \cdot 7 \\
24 \cdot 2\end{array}$ \\
\hline 11 & $5 \cdot 70$ & $\begin{array}{l}150 \\
200 \\
250\end{array}$ & $\begin{array}{l}34 \cdot 1 \\
34 \cdot 9 \\
35 \cdot 4\end{array}$ & $\begin{array}{l}36 \cdot 5 \\
37 \cdot 8 \\
39 \cdot 6\end{array}$ & $\begin{array}{l}15 \cdot 8 \\
14 \cdot 8 \\
12 \cdot 9\end{array}$ & $\begin{array}{l}13 \cdot 6 \\
12 \cdot 5 \\
12 \cdot 1\end{array}$ & $\begin{array}{l}0.538 \\
0.532 \\
0.502\end{array}$ & $\begin{array}{l}0.680 \\
0.695 \\
0.705\end{array}$ & $\begin{array}{l}27 \cdot 0 \\
26 \cdot 7 \\
25 \cdot 2\end{array}$ \\
\hline & $6 \cdot 62$ & $\begin{array}{l}150 \\
200 \\
250\end{array}$ & $\begin{array}{l}36 \cdot 2 \\
37 \cdot 1 \\
37 \cdot 6\end{array}$ & $\begin{array}{l}33 \cdot 6 \\
34 \cdot 2 \\
35 \cdot 8\end{array}$ & $\begin{array}{l}15 \cdot 8 \\
14 \cdot 8 \\
12 \cdot 9\end{array}$ & $\begin{array}{l}14 \cdot 4 \\
13 \cdot 9 \\
13 \cdot 7\end{array}$ & $\begin{array}{l}0.540 \\
0.532 \\
0.500\end{array}$ & $\begin{array}{l}0.680 \\
0.697 \\
0.707\end{array}$ & $\begin{array}{l}28 \cdot 8 \\
28 \cdot 3 \\
26 \cdot 6\end{array}$ \\
\hline
\end{tabular}


M.AY 1915. HEAT DISTRIBUTION IN GAS-ENGINE CYLINDER.

TABLE 5

Heat Balance at end of Expansion Stroke.

FULe LOad.

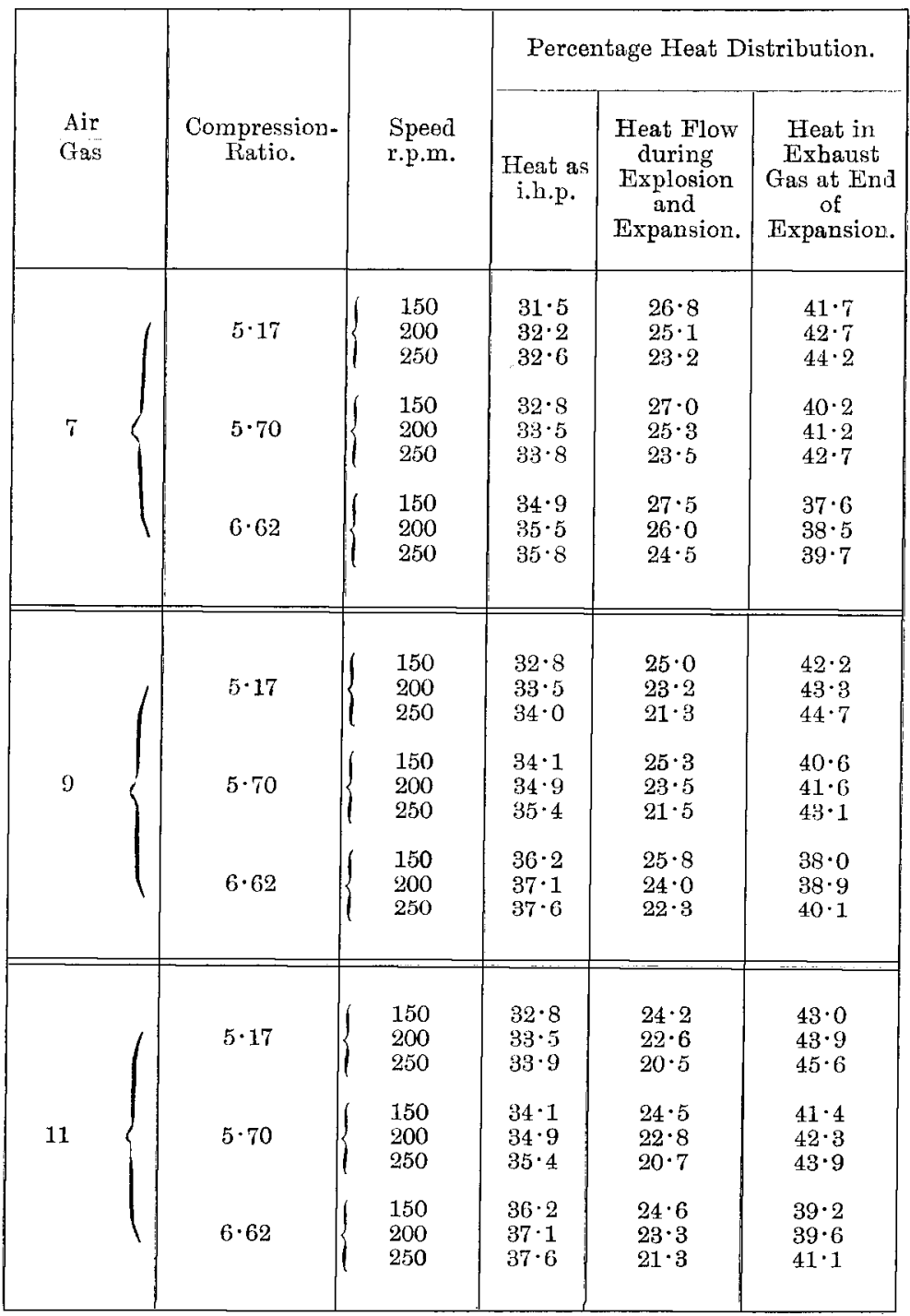




\section{Discussion in London.}

The President, in proposing a hearty vote of thanks to the authors for their very interesting Paper, said that a large amount of research had been made on gas-engines, especially on engines of about the size of that with which the authors had experimented; but there were still gaps in our knowledge, and the authors had certainly made a step in bridging at least one of those gaps.

The Resolution of thanks was carried with acclamation.

Dr. Dugasd ClerK (Member of Council), in opening the discussion, congratulated the authors on a very useful piece of work which filled up, as the President had said, a gap in their knowledge on the subject. As the members were aware, he (Dr. Clerk) had interested himself for a very long time in everything connected with gas-engines. His earliest attempt at such an analysis as was given in the Paper, on the heat distribution in the cylinder, was dealt with in a Paper he read at the Institution of Civil Engineers in 1882. In the earlier days of work on internal-combustion engines, he studied the heatlosses incurred in closed vessels, using gaseous explosions with different proportions of gas and air. Experiments of that nature made as far back as 1886 gave interesting data enabling one to predict what sort of heat-loss would be obtained in large cylinders as compared with the small explosion vessel used for experimental purposes. In those days it was generally considered that if a mass of flaming gas were exposed to the interior surface of a chambera closed vessel without any moving piston-that given equal density and equal mean temperature, the loss would be proportional to the time of exposure-that is, if there was double the time of exposure of a gaseous explosion to cold walls, double the loss would be obtained. That idea was broadly true in cylinders having no pistons, namely, vessels of a constant volume.

When experiments on actual engines began to be made, it was 
expected that, if an engine ran at 100 revolutions per minute, and if the same engine then ran at 200 revolutious per minute, the conditions of the diagram, the flame temperature, and so on being exactly the same, the heat-losses would be very greatly diminished; they would be halved at 200 revolutions per minute, because the time of exposure was halved. It was very soon ascertained that this was not the case ; in fact, under almostall conditions of running in actual engines, either in gas-engines or in petrol engines, the heat-losses seemed to change very little with the speed. Instead of diminishing, the rate of heat-loss certainly increased with the speed, and increased so much that it left very little improvement in the economy due to a higher speed. There was a speed in the engines where all the conditions (the conditions of the shape of the diagram more than anything else) gave the best results. In that case if the speed were increased, the rate of heat-loss was diminished so slightly that the expected economy was not obtained; the expected reduction of heat-loss was not forthcoming. For a long time that result was extremely puzzling; but some years ago several of those who were working for the British Association Committee on Gaseous Explosions, especially Professor Hopkinson and himself, found that the heat conductivity or the heat-loss in a cylinder varied very much with the rate of revolution. They made a number of experiments which seemed to show that the faster the engine ran, the greater the velocity the entering charge became in the cylinder, so that at a high speed it was easily possible to have an entering velocity as high as 150 feet per second. The idea among several of them was that that velocity persisted during the suction stroke and the compression stroke, and that when the engine was running fast the residual velocity movement in the cylinder at the moment of completion of compression was greater the higher the speed became. It was difficult to prove that that was actually so, but some experiments had now been made with an engine in which the valves were so arranged that the charge could be trapped. A charge could be taken in on an engine running at a certain speed, the charge trapped, and then the ignition prevented from occurring until the piston had moved two or three times out and in. In that 
(Dr. Dugald Clerk.)

way time was given for the turbulent air to still down. Under those circumstances it was found that the ignition varied greatly. The ignition became much slower when the charge was trapped in that way than in the usual way.

Then Professor Hopkinson tried some other experiments, also with an engine. In that case he used the trapping device, but he measured the rate of heat-loss without putting in any gas at all, using air alone. He used a heated platinum wire and measured the rate of heat-loss, and he found that the faster the engine ran, even when the platinum wire was only opened at the end of the compression stroke, turbulence of the gases caused the rate of heat-

FIG. 7.

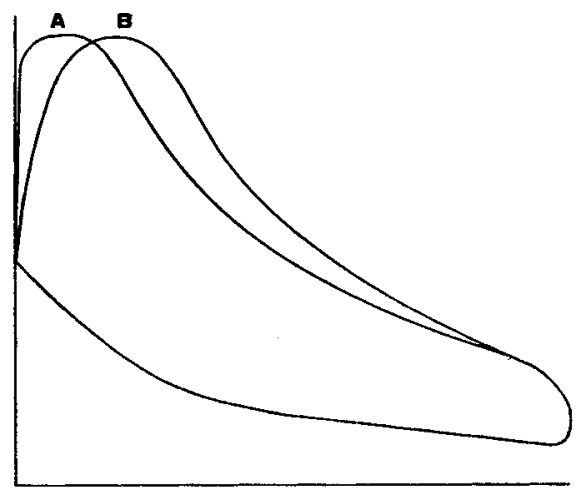

loss to increase with the rate of revolution of the engine. That at once showed that there was something in the nature of residual turbulence due to the inlet velocity of the charge. As far back as 1882 it would be found, from one of his old Papers, read at the Institution of Civil Engineers, that he was clearly conscious that there was a turbulence due to flame projection into a cylinder, but he was not conscious that the turbulence of the admission lasted to the completion of compression. Hopkinson found in addition that, if a fan were put in a closed vessel, the rate of cooling of the flame could be greatly increased by working the fan rapidly. He obtained velocities that represented the velocities in the cylinder, and identical heat-losses were also obtained. 
The present Paper was exceedingly valuable, because it investigated a matter that others had not fully studied, namely, from the end of determining the variation of heat-loss due to variation in the rate of revolution, which was a very difficult matter to determine. He congratulated the authors on the care they had taken in that respect, and the care also they had taken with regard to the shape of the diagram. For an experiment of such a nature, it was necessary to be extremely careful not only that all the other conditions were absolutely right, but that different conditions of diagram were not obtained.

If an ordinary gas-engine diagram something like the diagram $\mathrm{A}$, Fig. 7, was obtained, with a slightly rounded top, if in passing from one experiment to another, and from one speed to another, the same mixture was used and the rate of ignition was not altered, the diagram would change to $B$. The theoretical efticiency changed at once, so that a factor was introduced in which the added loss produced was not due to any change in the conditions of the cylinder, but the change in the position of the ignition-line. $\mathrm{He}$ noticed that the authors were careful about that, because they spoke in the Paper of keeping the ignition-line always straight. He presumed the authors never allowed the line to run forward in the manner shown in the diagram he had drawn.

Mr. W. J. WALKER said the authors always took the precaution to obtain a vertical explosion-line.

Dr. Dugald Clerk said that was very important, because a comparison absolutely depended on the lines being kept practically the same. The authors had given them a series of most interesting results from that point of view.

Another interesting point in connexion with the Paper was that, in most determinations of heat distribution in a cylinder, the jacket-loss--the heat flowing from the combustible gases, the burning gases, to the water-jacket--included not only the heat which flowed during the explosion and expansion to the moment of exhaust, but the very considerable quantity of heat which also flowed from the exhaust gases into the jacket under the 
(Dr. Dugald Clerk.)

exhaust-valve. He believed he (Dr. Dugald Clerk) first pointed out that cause of difficulty in a Paper which be read to the Institution of Civil Engineers in 1907, where he emphasized that to avoid this difficulty it was necessary to isolate the two waterjackets. He wished to point out that the authors in getting their engine made had the exhaust-valve part isolated from the rest of the water-jacket. It would be found that a description was given on page 364 showing that the exhaust-valve and the exhaust passage illustrated on the right-hand side of the diagram were surrounded by water, but were isolated from the water-jacket part $B$, so that in the A part they only obtained the small amount of heat which flowed through the cylinder-walls above the exhaustvalve, and a little to the left of the exhaust-valve in the vertical part they missed all the heat that passed into the jacket $A$, which was the heat that passed after the valve was lifted, when the gases passed under the valve and struck against the exhaust passage. In that way it was possible to isolate that portion of the heat, which should not appear in the balance-sheet at all. This appeared as heat lost to the sides of the cylinder, whereas it was heat lost after the moment of the opening of the exhaust-valve.

There was only one other remark he desired to make in reference to that experiment, namely, that it was rather difficult to isolate the loss in that way entirely. To be quite accurate, no heat should flow in through the exhaust-valve, and no heat should flow through the part near the exhaust-valve, only the heat coming after the exhaust-valve opened to make that a very good means of discriminating. He found, however, with much interest, that the authors arrived at a figure which he (Dr. Dugald Clerk) deduced from some of his own experiments with a peculiar type of diagram, which he called a zigzag diagram. He deduced from his own and from Professor Burstall's experiments that if the whole jacket-loss as determined in an ordinary engine were taken, about one-third of the loss was due to heat trapped under the exhaust-valve, so that where there was a jacket-loss in the old way of about 30 per cent., about 10 per cent. of it-the part going into the exhaust-yalye or into the wrong place-was obtained in 
that peculiar method. He noticed from page 379 that on the line of revolutions 200, which was the normal speed of the engine, with different quantities of air and gas, the amount of heat passing into jacket $B$ was roughly 10 per cent., and that was not very far out from what was found to be the number deduced in the other way. That number, however, must be a little high. He believed the other, the third, was also high, for reasons he had previously discussed. Taking all the data given in the Paper, one who had been in the habit of experimenting on such subjects could appreciate the amount of work that the investigators had put into it, and he was very grateful indeed to find there was a great number of figures upon which it was possible to reason further.

There was one interesting point upon which it was possible to reason definitely, namely, it was possible to get from those determinations a fairly good idea of the specific heat of the different gases used in the engines. $\mathrm{He}$ presumed the total exhaust-heats were taken from the exhaust condenser.

Mr. W. J. WALKER said they were; they were taken from the cooler.

Dr. Dugald Clerk asked if all allowances had been made.

Mr. WALkER replied in the affirmative.

Dr. Dugald Clerk said that in that case, in all those figures where the total exhaust-heat on the charge was taken, it was possible to make further deductions. It was possible to compare that method of determination of the specific heat of the actual gases used in the engine with the rather more abstract methods which had now been used both on the Continent and in England in determining the specific heat of those gases and not in the engine cylinder at all. He thought the experiments he described some years ago at the Royal Society were the only experiments that had been made where the determination of the specific heat was made in the actual cylinder, but the authors' figures gave another method of making the same determination, and should be very interesting. 
Professor H. Hubert (Liége University) thought the Paper was one of the most valuable that had been written in the last few years on the subject of the distribution of heat in gas-engines. He admired the way in which the authors had condensed so much valuable information into so small an amount of space. He was quite sure that if the experiments had been carried out by engineers of another nation, they would have been explained in a large book with many references to other works, and the conclusion that these engineers alone knew the question.

He was glad to find that the authors had verified in their experiments the exactitude of a law which was not absolutely exact but nearly so, namely, that for a given engine working at full load the amount of heat lost by the exhaust gases and by the jacket was nearly constant. Professor A. Witz and himself had enunciated that law in the course of some experiments they had made on large gas-engines at the Cockerill Works. They had not tried it at different speeds, but they had tried it with reference to the temperature of the water entering the jacket, and found that there was hardly anything to be gained, because when one of the lossies was diminished the other was increased, so that the sum remained constant. He found on referring to Fig. 5 (page 372) that the sum of the two losses at the two ends of the diagram was exactly the same, and it was the same also in Fig. 3 (page 370). It was interesting to know that it was very difficult to gain anything by increasing the speed, the number of revolutions per minute, and the brake horse-power. He and his colleague had found the same law in raising the temperature of the water issuing from the jacket.

He noticed from the Paper that the authors had measured the temperature of the expansion, not experimentally, but by two calculations, on the supposition that the gases only obeyed the general laws in regard to perfect gas. He wished to remind the members that one of his assistants, Mr. Duchesne, had measured exactly the temperature of the walls and of the steam in a steamengine with very accurate thermo-electric thermometers, measuring the temperatures at each one-tenth of the stroke of the piston. 
Without in any way wishing to criticize the method adopted by the authors, he suggested that it would perhaps be possible for them to have an exact measurement made, with Mr. Duchesne's thermo-electric thermometer, which had been described in the Revue Universelle des Mines, ${ }^{*}$ the official publication of the Association of Engineers of the School of Liege. He hoped the authors would endeavour to secure the results of calculation by a direct measurement of the temperature at the end of the expansion.

$\mathrm{He}$ wished also to point out that, according to recent and unpublished experiments made in the laboratory of the Fabrique nationale d'armes de guerre (F.N.) at Herstal, near Liége, the thickness of the walls of a gas-engine had no sensible action on the wall-loss. Two engines, differing only by this thickness, which was in one case $7 \mathrm{~mm}$. and in the other $25 \mathrm{~mm}$., had nearly the same thermal efficiency of $18 \cdot 5$ and $18 \cdot 8$ per cent.

Mr. E. J. Davis desired to inquire whether the following statement made by the authors on page 376 was quite correct: "Since at 150 revolutions per minute the period of contact per cycle, of hot gases and cylinder walls, is 1.66 times as great as at 250 revolutions per minute, the rate of transmission of heat through the cooling surfaces is evidently much greater at the highest speed." Although the engine was running faster, was the speed for the working stroke in that proportion faster, because the cyclical variation of that engine at 150 revolutions would be considerably more than the cyclical variation of it at 250 revolutions, so that the time taken for the actual working strokes would certainly not be $1 \cdot 66$ times at the lower speed? The effect of this cyclical variation tended to lessen the difference in times of exposure of working fluid at the two speeds, decreasing $1 \cdot 66$ to some small extent. It was to be regretted that the indicator diagrams taken at the various tests were not given in the Paper, as these would have enhanced the practical interest. The backpressure of the exhaust at the two speeds, if the valve-settings were the same for both tests, would affect the mixture and 
(Mr. E. J. Davis.)

temperature of the charge before ignition, tending to make the charge more inflammable at the higher speed. An extreme case of the effect of exhaust gases he (Mr. Davis) cited in the discussion upon Professors Coker and Scoble's Paper at the Institution of Civil Engineers on "Cyclical Changes of Temperature in a Gas-Engine Cylinder."

There was no evidence that the percentage of exhaust gases in the charge was higher at the higher speed, but if the settings were correct for 150 revolutions per minute, unless there was no backpressure at the end of the exhausting stroke, it was difficult to understand how a homogeneous mixture could be obtained at both speeds. If, on the other hand, the first third of the diagram were taken and compared with the first third of a diagram taken at 150 revolutions per minute, and the average pressure for that portion was found greater at the higher speed, it would tend to show that the charge was more inflammable at the higher speed, in spite of the fact that a higher initial pressure was obtained at the lower speed. If the greater percentage-loss at the higher speed were due to turbulence of the fluid, one would expect the charge before ignition to be more inflammable and to increase the initial pressure, in spite of the higher speed, but from experience it was known that this was not so. Did turbulence before ignition have a marked effect upon the fluid after ignition, considering the fierce exchanges that must be taking place? The film of oil on the walls of the cylinder must have some effect upon the passage of heat. Were the conditions from this effect identical at both speeds?

Captain H. Riall Sankex (Member of Council) pointed out that the top curve in Fig. 3 (page 370) was called " Efficiency Ratio on I.H.P." That ratio had been obtained by dividing the thermal efficiency of the actual engine by that of the corresponding airstandard as defined by a Committee of the Institution of Civil Engineers, and was called the "Relative Efficiency" by that Committee. The air standard was based on pure air, assuming constant specific heat and taking a value for $\gamma$ of $1 \cdot 4$. This standard was taken because at that time the knowledge of the 
variation of the specific heat of gases was imperfect. Later on, due very much to the work of Dr. Dugald Clerk, Professor Hopkinson, and others, the law of the variation of the specific heat with temperature was determined, with sufficient accuracy to calculate the thermal

FIg. 8 .
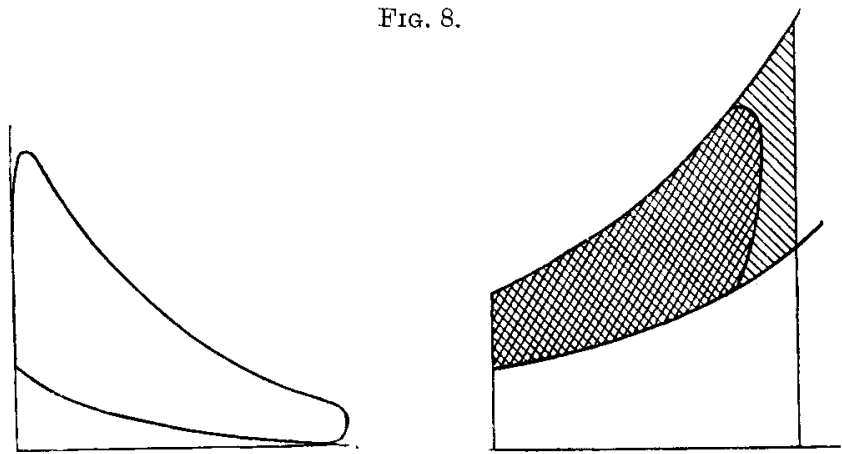

efficiency of an ideal engine using the working substance-that is, the product of combustion-and in the discussion on "The Third Report of the Gas-Engine Research Committee," Dr. Dugald Clerk gave a curve showing the thermal efficiency of an ideal engine using the actual working fluid for various ratios of compression. Thus for a

FIG. 9.
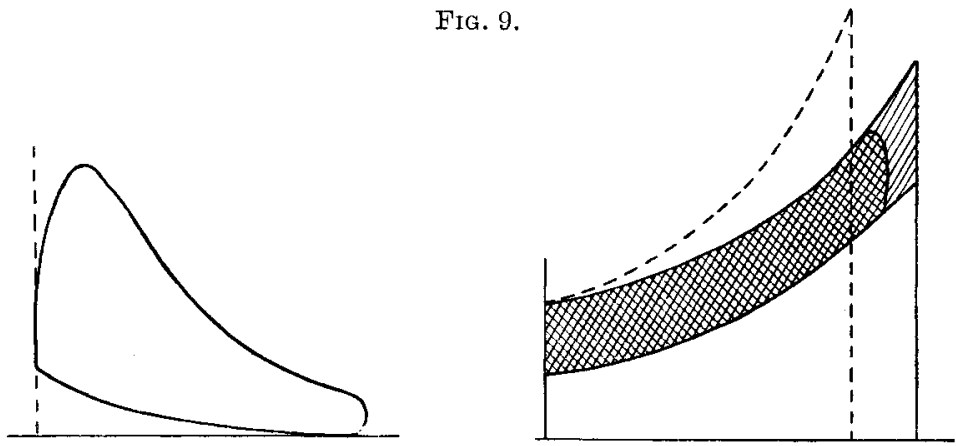

$5 \cdot 17$ compression (the compression in the trials, of Fig. 3), the efficiency was 39 per cent. instead of 48 per cent. for the corresponding air-standard. From Fig. 3 it would be seen that the thermal efficiency of the actual engine for the trial at 30 b.h.p. was 
(Capt. H. Riall Sankey.)

$33 \cdot 5$ per cent., and hence the ratio between what the engine itself did and what an ideal engine could do using the same working fluid was $\frac{33.5}{39 \cdot 0}$, or $0 \cdot 85$. In the discussion on Dr. Dugald Clerk's Paper, to which Dr. Dugald Clerk had just referred, he (Captain Sankey) suggested that the expression "efficiency ratio" should be employed when the comparison was made with an ideal engine using the working fluid, in the following words: "He would suggest that 'relative efficiency' might be retained when comparing a gas-engine with the air standard, and 'efficiency ratio' should be used when comparison was made with the actual standard." Based on that, he suggested to the authors that they should also plot the "efficiency ratio" as above defined, and alter the words "efficiency ratio" given in the Paper to "relative efficiency."

Dr. Dugald Clerk had stated that in Diagram A, Fig. 7 (page 388), a better thermal efficiency was obtained than in Diagram B. The reason for this could be shown by the $\theta \phi$ diagrams, given in Figs. 8 and 9, which corresponded to a vertical and sloping explosion-line respectively. In each diagram the cross-hatched area represented the heat utilized by the actual engine, and it was obviously less in the latter diagram.

Mr. W. A. TookeY said he quite agreed with all that had been said in regard to the value of the Paper; it was one that could not be fully digested or even properly appreciated in the short time that the members had had the printed copy before them. He desired to ask a question in connexion with the calorific value given for town gas on page 367. The determination of calorific value was of great importance to manufacturers and others interested in gas-engines. It had hitherto been rather difficult to obtain a close comparison between calorific values determined by a calorimeter and those computed from figures. He noticed in the Table given by the authors it was stated that: "The corresponding lower calorific value (calculated) at standard temperature and pressure is 521.4 B.Th.U. per cubic foot. . . The mean of the lower and higher values obtained during the trials were respectively 
520 B.Th.U. and 577 B.Th.U. per cubic foot." It was quite evident that the authors had obtained a very close comparison between those two methods, one a computation and the other a measurement. When the Paper was finally printed he hoped the authors would insert the values they had taken for the different constituents that made up the total volume of the fluid. Using the values that were to be found in text-books upon the subject, and which had been based upon figures like Berthelot's, the calculated figure on the lower scale, for a gas of the composition mentioned in the Paper, would come out considerably less than the figures given by the authors, and he therefore suggested that in the final print of the Paper it would be interesting and valuable if the multipliers which the authors had used were added.

He thought it was a pity that the question of the different strength of mixtures was referred to only in the proportion of so many volumes of air to gas. A very much more useful comparison could be made when the figures were given on the actual number of B.Th.U. in a cubic foot of the working mixture entering the cylinder. Not only that, but knowing the ratio of expansion or compression to the engine-that is, the volume before compression to the volume after compression-one could compute the mixture strength of the working charge, taking into consideration the compression volume as well. That method of comparison gave an entirely different point of view. He had found personally that, using that basis, comparisons of tests gave a very useful rneans of comparing not only the performances of one engine, but the relative performance of many engines of different types yorking with gas and with other classes of fuel under various conditions. It was that universality or ability to compare engines operating with the different classes of fuel that was of considerable value to internal-combustion engineers. It might be remembered that, in the early part of the previous year, it was his privilege to read a Paper before the Institution upon "Commercial Tests of Internal-Combustion Engines." He then suggested that method of referring to the calorific value of the charge, and pointed out how on that basis it was possible to compare and to obtain a ratio 
(Mi. W. A. Tookey.)

between the mean effective pressure produced to the heat entering the cylinder. That factor, as was pointed out by Captain Sankey at the time, could be used in another way for the determination of temperatures. It could also be used in another way. Knowing the strength of mixture, and knowing the factor for any particular compression-ratio, it was possible to obtain by calculation not only the mean effective pressure, but the useful pressure referred to the brake horse-power, and also, by difference, the pressure that had evidently been dissipated in overcoming the mechanical and fluid resistances of the engine.

For example: from Table 4 (page 384) the following comparisons were made possible:-

$\mathrm{F}$ (Tookey factor) $=\mathbf{E}_{t h}$ (Thermal efficiency i.h.p. or b.h.p.) $\times$

$$
\frac{\mathrm{R}}{\mathrm{R}-1} \times \mathbf{5} \cdot 4
$$

and for

$$
\mathrm{R}=5 \cdot 17 ; \mathrm{F}=\mathrm{E}_{t h} \times 6 \cdot 7 \text {. }
$$

\begin{tabular}{|c|c|c|c|c|c|c|c|c|c|}
\hline \multicolumn{3}{|c|}{$\frac{\text { Air }}{\text { Gas }}=7$} & \multicolumn{7}{|c|}{$\begin{array}{l}520 \text { B.Th.U. in } 8 \text { c. ft. of mixture, and } 65 \text { B.Th.U. per } \\
\text { c. ft. of mixture admitted, equivalent to } 65 \times \frac{4 \cdot 17}{5 \cdot 17}= \\
52 \cdot 4 \text { B.Th.U. per c. ft. of total cylinder volume. }\end{array}$} \\
\hline \multirow{2}{*}{ घ्वे } & \multicolumn{2}{|c|}{ I.h.p. } & \multicolumn{2}{|c|}{ B.h.p. } & \multirow{2}{*}{$\begin{array}{l}\text { I.h.p. } \\
\mathrm{P}_{m} \text {. }\end{array}$} & \multirow{2}{*}{$\begin{array}{l}\text { B.h.p. } \\
\mathrm{P}_{n} .\end{array}$} & \multirow{2}{*}{$\begin{array}{l}\text { F.h.p. } \\
\mathrm{P}_{f}\end{array}$} & \multirow{2}{*}{ 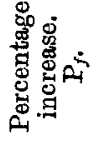 } & \multirow{2}{*}{ 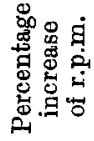 } \\
\hline & $\mathrm{E}_{t h}$ & F. & $\mathrm{E}_{t h}$ & F. & & & & & \\
\hline 150 & $0 \cdot 315$ & $2 \cdot 11$ & 0.277 & $1 \cdot 855$ & $110 \cdot 5$ & $97 \cdot 2$ & $13 \cdot 3$ & 1 & 1 \\
\hline 200 & 0.322 & $2 \cdot 155$ & $0 \cdot 274$ & $1 \cdot 835$ & $112 \cdot 8$ & $96 \cdot 2$ & $16 \cdot 6$ & $1 \cdot 25$ & $1 \cdot 39$ \\
\hline 250 & 0.326 & $2 \cdot 18$ & 0.256 & $1 \cdot 715$ & $114 \cdot 1$ & $89 \cdot 8$ & $24 \cdot 3$ & $1 \cdot 835$ & $1 \cdot 66$ \\
\hline \multicolumn{2}{|c|}{$\frac{\text { Air }}{\text { Gas }}=11$} & \multicolumn{8}{|c|}{$\begin{aligned} \therefore 520 \div 12 & =41.65 \text { B.Th.U. per c. ft. of mixture admitted, } \\
& =33.6 \text { B.Th.U. per c. ft. of mixture in total } \\
& \text { cylinder volume. }\end{aligned}$} \\
\hline 150 & 0.328 & $2 \cdot 195$ & 0.26 & $1 \cdot 741$ & $73 \cdot 8$ & $58 \cdot 5$ & $15 \cdot 3$ & 1 & 1 \\
\hline 200 & 0.335 & $2 \cdot 24$ & 0.257 & $1 \cdot 721$ & $75 \cdot 2$ & $57 \cdot 8$ & $17 \cdot 4$ & $1 \cdot 137$ & $1 \cdot 33$ \\
\hline 250 & 0.339 & $2 \cdot 27$ & 0.242 & $1 \cdot 62$ & $76 \cdot 2$ & $54 \cdot 4$ & $21 \cdot 8$ & $1 \cdot 425$ & $1 \cdot 66$ \\
\hline
\end{tabular}


From the presentation of figures in this form it was clear that, with a rich mixture, there was, with increase of speed, a higher percentage of power absorbed in mechanical and fluid friction than with weak mixtures, and, moreover, that it was not in direct proportion with speed. From other tests made by the speaker, fluid friction appeared to be more accountable for the differences noted than did mechanical friction, and it would be interesting to know, from a study of the light spring cards taken by the authors, whether this was confirmed or not by their experiments.

By calculations from the thermal efficiencies given in Table 1 (page 381) it was also possible, by means of the Tookey factor, to obtain the values tabulated below, and by further computation the number of impulses per minute were calculable, so that it became possible to determine the value of $\mathrm{P}_{f}$-or pressure absorbed in mechanical and fluid friction in "hit" cycles-and to correct it for the total number of cycles per minute which then rendered it comparable with the values of $\mathrm{P}_{f}$ in the Table on page 398 .

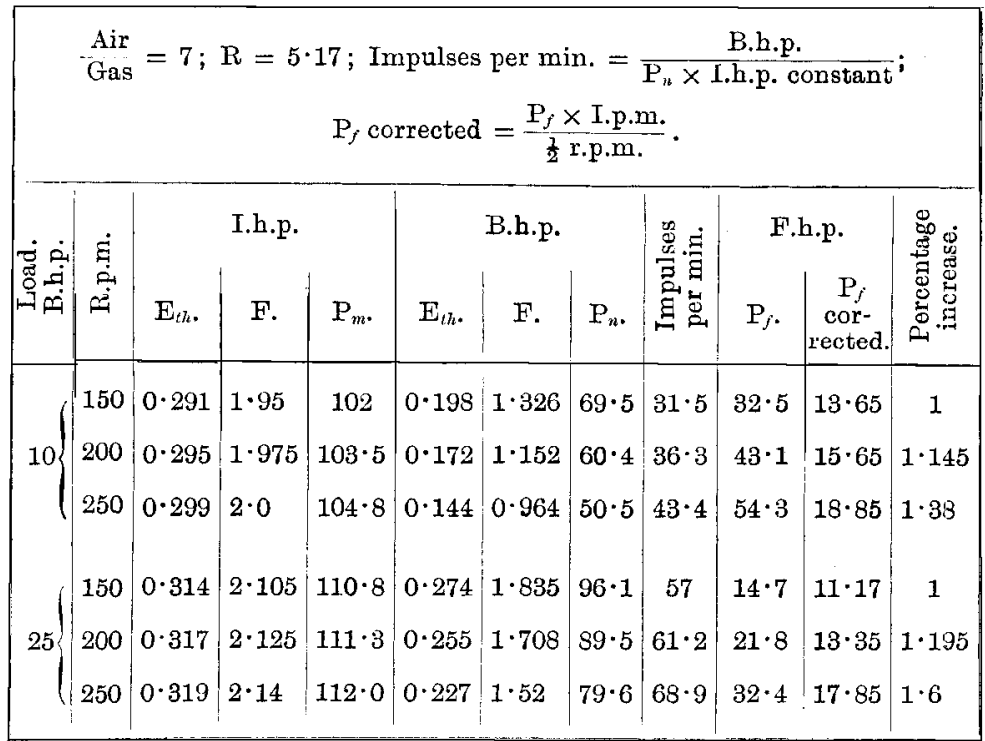


(Mr. W. A. Tookey.)

It would be evident from the foregoing that the increase of $P_{1 n}$ and number of impulses per minute reduced the corrected values of $\mathrm{P}_{f}$ from $13 \cdot 65$ to $11 \cdot 17$ at 150 revolutions per minute when the output increased from 10 to 25 b.h.p. How much of this was due to the fluid friction falling off in relation to the ratio of "hit" to "miss" cycles could no doubt be deduced also from the light spring cards.

The President said that the worst feature of very valuable Papers was that their authors had so worked at the subject that there was very little left to take objection to. The Paper was an extremely valuable one, and he was sure the authors would be pleased with the general testimony which had been given to the importance of the research they had made, which had been one involving a very considerable amount of labour.

Mr. W. J. WAIJKER, in reply, after thanking the members for the very kind way in which the Paper had been received, said he would like to illustrate the types of diagrams which the authors obtained at different speeds when making the tests. Taking an ordinary case of a very slow speed and rich mixture, a diagram was obtained which had a sharp point, as shown in Fig. 10.

At the other extreme, when working with high speeds and weak mixtures (which tests were the most difficult to carry out), a diagram of the type shown dotted in Fig. 10 was obtained. The high speed and weak mixture gave a vertical line with a rounded top; it was impossible to get a sharp diagram under such conditions. When working at a speed of 250 revolutions per minute with a weak mixture, the engine sometimes stopped after running for about 5 or 10 minutes, owing to the weak explosions. Although some difficulty was experienced, the authors persevered, and possibly, out of about twenty tests at the highest speed and weakest mixture, half that number were obtained lasting from 20 to 30 minutes. The diagrams shown also illustrated the statement made on page 376 , that at the high speeds the average temperature during the stroke was about 6 per cent. less than it 
was at the slow speeds, and that accounted for the slight difference in the heat transmission to the jackets.

Attention had been called to the statement made on page 376 . The words were there used: "With the strongest mixture, and at full load, the percentage heat transmitted to these jackets is $1 \cdot 10$ times as great at 150 revolutions per minute as at 250 revolutions per minute, while with the weakest mixture the ratio becomes $1 \cdot 23$." Turning to page 366 , it would be seen

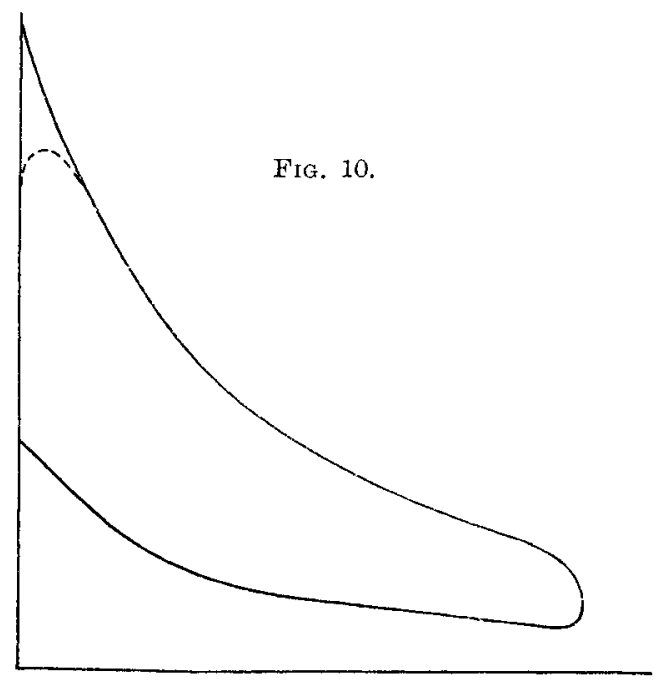

that the full load varied almost directly as the engine's speed. Full load was not a constant quantity with regard to speed. If it varied almost directly as the speed, then the energy involved per cycle must be the same in each case at both speeds, high and low, so that the quantity considered as constant on page 376 was not the load but the energy per cycle. It would be noticed that the statement was made on page 376 : "With the strongest mixture and at full load." Although it might seem that the increased percentage heat transmission was due as much to increased load at higher speeds, yet such was not the case. When considering the 
(Mr. W, J. Walker.)

effect of increased load upon percentage jacket-losses, it must be remembered that the increased load was obtained by decreasing the number of missed explosions, which was not the same thing as increasing the speed. Supposing they had the same energy per cycle at the different speeds, then the time of contact per cycle of the hot gases with the cylinder walls at 250 revolutions per minute was 0.6 times that at 150 revolutions per minute. The percentage heat-loss, assuming it to depend directly upon time of contact, should therefore be 0.6 times that at 150 revolutions per minute. As a matter of fact it was 0.91 and 0.813 in the two cases considered. Taking the slightly diminished temperatures at the higher speed into account, these two values of 0.91 and 0.813 would approximate to unity, hence the effective conductivity of the gases must have been increased in proportion to the speed.

On the other hand, taking two cases of load variation, say full load and three-quarter load at the same speed, it would be found that there was a diminished percentage jacket-loss at the lower load. That could not be ascribed to a shortened period of contact, as in the case of the difference in speed, but it was due to the cooling action of the air admitted during the missed explosion cycles, a condition which did not hold in the case of full load at different speeds.

Mr. Davis had pointed out that the cyclical variation of the engine speed at 150 revolutions per minute would be considerably more than the cyclical variation at 250 revolutions per minute, and that the effect of this would tend to lessen the difference in times of exposure of the working fluid. This was certainly true if the slower speed had been obtained at the expense of more irregular running. The comparisons made, however, were all at full load, and under such conditions the cyclical variation in the engine speed at different speeds must have differed very little. At lighter loads the cyclical variation in speed at the lower speeds was appreciably greater than at the higher speeds, but, then, no comparisons had been made between such cases. Any difference between the cyclical variation at full load and different speeds, if it did affect the question, must affect it to a small extent only. 
The arrangement of jackets was a design of Professor Gibson's intended primarily for experimental purposes, and it was very instructive indeed to have the exhaust jacket-losses separated in that way. He would be very pleased to carry out Captain Sankey's suggestion, and alter "Efficiency Ratio" to "Relative Efficiency." * He intended to work out the actual values of the efficiency from the properties of the real working fluid, and it would be interesting to see the difference between the two. He would here mention that the $\frac{\text { air }}{\text { gas }}$ ratios were obtained from the meter readings, so that the actual figures of $7:\{1,9: 1$, and $11: 1$ would be slightly larger, due to the dilution of the mixture by a portion of the exhaust products. In conclusion, he again desired to thank the members, on behalf of Professor Gibson and himself, for the kind manner in which the Paper had been received.

\section{Discussion in Manchester.}

The Chairman (Professor J. E. Petavel) said he was expressing the view of all present in stating that they were much indebted to the authors for bringing the matter before the Institution. 'The careful study of the internal-combustion engine was, of course, a subject of great importance. Professor Gibson was occupied with gas-engine research some four or five years ago when he was in Manchester, and he had had a very long experience of such experimental work. The internal economy of the gasengine was extraordinarily complex, and to some extent many of the usual terms were liable to error. The errors of the indicator card had been discussed time after time, and everybody was in agreement as to the difficulties which arose in that direction. Another kind of difficulty arose from the large differences in socalled mechanical efficiency found by various people, but those

\footnotetext{
* See footnote on page 370.
} 
(Professor J. E. Petavel.)

discrepancies were largely due to the fact that the mechanical efficiency of a gas-engine could be made to vary to a very considerable extent by altering what did not belong to the gasengine at all, but was simply in accidental relation to it, namely, the connexions, the exhaust-pipes, the gas-bag, etc.

Since Professor Gibson left Manchester they had been engaged in the Whitworth Laboratory on work in connexion with the gasengine, and in conjunction with Professor Asakawa, who came from Japan to carry on such work, they had made a complete analysis of the losses. He thought it might add something to the discussion if he brought before the Meeting the result of this analysis. The first thing to remember was that, as was well known, only a part of the total heat was available, and that the system of comparing the efficiency of the gas-engine with that of the air-engine was purely artificial, for even a theoretically perfect gas-engine could only reach about 80 per cent. of air standard. This limit was fixed by the fact that the working fluid used in a gas-engine was not air, but a mixture containing $\mathrm{CO}_{2}$ and $\mathrm{H}_{2} \mathrm{O}$, and that the specific heat of such a mixture was not constant but variable. When that was fully understood, they came to the conclusion that the margin of inefficiency remaining was very small. A study of the jacket-losses could not in itself throw much light on this question, because a heat loss through the jacket of, say, 5 per cent. or 10 per cent. occurring at the beginning of the expansion would be a loss of 5 or 10 per cent. in the efficiency; a loss of 10 or 100 per cent. occurring at the end of the expansion would be no loss at all in the efficiency. So it depended where the heat was lost.

He would now say something with regard to the question of the relative importance of the various losses. An ideal engine working with a compression-ratio of $4 \cdot 85$, and having no thermal or mechanical losses, would have a thermal efficiency of 47 per cent. on the air-standard, but only $36 \cdot 7$ if it used the mixture actually employed in an ordinary gas-engine. The point at issue was, how nearly did the actual engine approach to that ideal? They could divide the losses into two parts: one representing the 
difference between the indicated h.p. of the actual engine and the indicated h.p. of the perfect engine, and the other representing what was usually put down as mechanical losses. The difference between the indicator card as drawn and that of an absolutely perfect engine was small, amounting only to about 10 per cent. of the latter, so that there was not much margin remaining. The engine behaved exactly as if 5 per cent. of the available power was lost before expansion-about a half per cent. during the explosion and 4 per cent. during the expansion. With regard to the mechanical losses, friction and valve-lifting represented about 11 to 12 per cent. of the total available energy, the windage of the fly-wheel about $1 \frac{1}{2}$ per cent., and the pumping losses about 7 per cent. About half the pumping loss was due to the resistance of the inlet and outlet pipes, and hence the mechanical efficiency as usually defined depended to some extent on a factor not directly connected with the design of the engine.

Such an analysis of the losses might perhaps be of some practical value to designers, although one must admit it showed that the commercial engine approached already so nearly to the perfect engine working on the Otto cycle that much further gain was hardly to be expected. It was possible that in the future the gas-engine might be worked on a cycle which would render available considerably more than one-third of the heat, but this was a fundamental change which could not at present be regarded as a practical proposition.

There was one other point he would like to refer to, namely, radiation. Radiation was a factor which served to cover the errors made in the estimation of all other thermal quantities, and, as usually expressed, it had very little to do with actual physical radiation or loss of heat from the surface itself. In the present case he saw that the temperature of the outside surface of the engine-cylinder was kept constant. The radiation from the cylinder ought therefore to be constant. He did not know whether the authors assumed that to be the case.

Mr. WALkER said that they did. 
The Charrman concluded by expressing the hope that the Paper would lead to a really profitable discussion.

Mr. E. G. HILler said he had the pleasure of knowing Professor Gibson before the latter went to Dundee, and always thought him a very able man, who, when be came to investigate these matters, would bring practical experience to bear on the subject.

When he heard the Chairman discoursing on the question of improvements in gas-engine design in the direction of efficiency, it seemed to him that the attention of the professorial gentlemen was turned in the wrong direction. He did not think the improvement of gas-engine design in the direction of efficiency was the most important matter at the present moment. The ordinary gas-engine was efficient as a heat engine but deficient as a reliable motor, and that was where the professors ought to give their attention. The search for efficiency in the sense used in the Paper might lead to incorrect conclusions as regards gas-engine construction and design. On Fig. 3 (page 370) it was shown that the mechanical efficiency, the thermal efficiency on i.h.p., the thermal efficiency on b.h.p., and the efficiency ratio on i.h.p., all increased with increasing loads. On the other hand, it had to be borne in mind that with increase of loads the reliability of the ordinary type of gas-engine, as measured by freedom from breakdown, decreased. Also on Fig. 5 (page 372), jacket-losses, and in Table 3 (page 383), radiation-losses, were shown to diminish as the speed increased. What, however, was the practical bearing of this effect? Was it desirable to reduce these losses in this way in a practical engine when the inevitable result of an increase of speed and increase of metal temperature was to increase the liability to mechanical breakdown?

Commercial competition had in recent years had a marked effect in this country in increasing the speed of revolution in the ordinary commercial type of horizontal gas-engine. Mr. J. G. Walthew read a Paper before the Manchester Association of Engineers last Session, in which he dealt with some of the more 
commercial questions touching the design and construction of the gas-engine. In the course of that Paper he referred to the higher gas-engine speeds in connexion with which it had been found necessary to put balance-weights on the cranks. The history of it was as follows. In the first instance, gas-engine makers supplied a so-called ordinary type of engine running at about 160 to 170 revolutions per minute or thereabouts, and they sold an electriclight type of engine which ran at a higher speed with balanceweights. Of course the higher speed engine with more strokes per minute, having the same power developed per stroke, was more powerful than another engine of the same size running at a lower speed, and that was a commercial or selling factor.

The result of this factor was to cause a general increase in speed of the ordinary horizontal gas-engine in this country. Running at a higher speed, however, had increased the unreliability of these gas-engines though accompanied by a lower relative first cost and possibly a greater degree of thermal efficiency.

He had allowed himself to deal with questions not directly touched by the Paper, because he thought the professors were on the wrong line. For instance, the combustion chamber at the back end, at which some of the heat losses discussed in the Paper were measured, was one of the things that gave much trouble by cracking. In his opinion it was a more important matter that they should learn how to prevent such cracking of the casting in the valve-passages, combustion chamber, and liner than that they should try to get one or two per cent. more thermal efficiency, and the professors would do more for the development of the gas-engine by making it equal in reliability to the steam-engine than they would by confining their attention to the single question of thermal efficiency. There was much room for investigation in the direction of the improvement, in detail, of those castings, and in the qualities of different mixtures of iron, which if properly carried out should result in distinctly better working appliances being obtained.

He would like to refer to a defect in the design of the ordinary 
(Mr. E. G. Hiller.)

gas-engine of commerce which was particularly objectionable in the larger sizes-namely, the adoption of the trunk form of piston. In the steam-engine it had been recognized for many years past that the cross-head was an essential part of the horizontal engine and it had a definite function to perform. In the large size of horizontal gas-engine the combination of the duties of piston and cross-head in the trunk piston led to objectionable results. It might be that with a cylinder of about 6 to 8 inches diameter the trunk piston was satisfactory, but when they came to cylinders of 16 to 20 inches diameter it was mechanically incorrect to throw on to the trunk piston the double duty of piston and cross-head, and this feature accounted for a large proportion of the cracked liners and other troubles which led to breakdowns in gas-engines.

The principal point which he desired to make clear was that investigations of the kind dealt with by the Paper were not directed to the most important of the questions which concerned the working of gas-engines. This criticism concerned not only the Paper before the Meeting, but also equally applied to others which had been published in relation to questions of a more or less academic character. The object of this expression of his views was to help in bringing about working improvements in the construction of the ordinary gas-engine and also to bring before the professorial investigators the desirability of dealing with the practical problems which concerned those who had, in their daily work, to deal with gas-engines and their failings and shortcomings.

Mr. H. N. Bickerton complimented the authors on the Paper, which included valuable information, though it did not include what Mr. Hiller was always asking for. Every engineer who paid attention to gas-engine construction desired that improvements should be made, and they would be very pleased to have the help of the professors in bringing them about. At the same time it was not fair to say that the gas-engine was not a reliable motor; be believed it would become the most reliable motor in existence. The number that were now being used, the growing demand for them, 
and the fact that manufacturers of them were very busy went to prove that statement.

Mr. AlfRed SAXON said he had prepared a few remarks on the general question of research work in connexion with engineering. The authors were to be congratulated upon the results of their investigations, which appeared to have been carried out in a careful and scientific manner. Their experiments, when studied, would go to prove how far the present gas-engine practice was right, and in what direction improvement might be obtained. As engineers, they ought to appreciate all research work and encourage it in connexion with whatever branch of the engineering industry it was undertaken, because it was evident that after a certain stage of development had been reached with any machine or prime mover, it could only be brought to a higher state of development by careful research work. The British engineers, he felt sure, had some leeway to make up, and if they did not carry out the necessary investigations, the foreigner would do it for them and reap the benefits. He wished to see the gas-engine made into a more perfect machine, if that were possible, than at present. His experience was that they found all kinds of prime movers required overhauling and repairing, and if one were guided in that matter by the experience of insurance companies (these remarks were prepared before the discussion that had taken place that evening), there was a much greater proportion of breakdowns and accidents in internal-combustion engines than in prime movers where steam was employed. He thought he was quite correct in saying that the area covered by the Manchester Association of Engineers formed the largest gas-engine manufacturing centre in the world, and any improvements in the gas-engine must have its effect on that large branch of the engineering industry of the district.

In concluding his remarks, he would like to call attention to one point in the Paper. In the comparisons the authors had made between the Hopkinson experiments and their own, the speed of the Hopkinson engine was given as 110 revolutions, whereas the speed of the authors' tests ranged from 150 to 250 revolutions. 
(Mr. Alfred Saxon.)

Could any difference in results be accounted for by the difference in the speeds?

Mr. Georae E. Windeler (Stockport) said he was not connected with gas-engine manufacture, but he had something to do with the internal-combustion engine, his branch of it being the Diesel oil-engine. They were much indebted to the authors for the information they had given in their researches. The bearing these researches had on the subject, so far as manufacturers were concerned, was this, that all such investigations led them on to a new line of thought as to how they might approach the difficulties which were frequently experienced. The cracking of breech-ends or pistons on heat-engines had been a difficulty all engineers had to face from time to time, and the results of the authors' experiments tended to show how to approach the subject from another point of view and lead to further investigations of the troubles. In Manchester, which was the home of the gas-engine industry of the Empire, there were men who could tackle most problems as well as anybody abroad, and it was necessary, in his opinion, to approach the matter from the professors' point of view. Engineers had to look at it from a monetary point of view, but the professors had not, and they could help forward the investigations in many ways. Naturally, insurance companies approached the subject from their own point of view. They had to provide for cracked pistons and cracked cylinder-covers; that was why engineers insured their engines-so as to be released from financial liability in case of breakdowns. Engineers were always open to receive suggestions, and if insurance companies would take up some form of research work as a means of assisting the industry to overcome the difficulties they had to face, it would be to the advantage of the insurance companies.

Mr. Frank Foster said that, although he had not intended to contribute to the discussion, it was turning in a direction which made him desire to say a few words. He appreciated work such as the authors described in the Paper. Before they could make 
substantial progress with gas-engine design they must know exactly what thermal reactions were taking place inside the cylinder. When they knew where the heat went to, the next step would be to ascertain the wall temperatures; and when they had got the temperatures and the hent flow for the principal parts of the cylinder, they were not a long way from having definite information on which to modify and improve mechanically the designs now existing.

He wished to refer, in passing, to Fig. 5 (page 372), where there was something not exactly a mistake, but certainly misleading. The mechanical efficiency curve dropped very considerably as the speed increased. That was probably quite true of the engine tested, but it was not true of engines designed for different speeds; that was to say, if an engine designed for 150 revolutions per minute had a mechanical efficiency of 82 or 83 per cent., another engine properly designed for 250 would have a mechanical efficiency closely similar. He imagined the drop shown in Fig. 5 was dur to extra fluid friction losses at increased speeds, mainly arising from valve-passages designed probably for the lower speed. At any rate one did not find in practice that the high-speed engine was specially low in mechanical efficiency.

Some engines he knew of were originally designed for a high compression, and on test they were found to consume about 8,800 B.Th.U. per b.h.p.-hour. That was a very low figure--in fact, probably a record for very large engines. Signs of trouble were noticed, and it was thought desirable to reduce the compression. It was reduced very largely--too much so in fact-and the consumption went up to approximately 10,000 B.Th.U. per b.h.p.-hour. For many years the proprietors ran the engines at that compression and with that gas consumption, because it gave them lower rates of heat transmission to the cylinder and piston walls-lower wall temperatures generally-and allowed improved working and lower overall costs. Later on they concluded that they had reduced the compression too far. It was somewhat increased and the gas consumption reduced. Those practical working results, he thought, had a direct connexion with 
(Mr, Frank Foster.)

investigations such as the authors had made. He would like to suggest that, with the investigation of heat distribution, the authors should couple an attempt to estimate the temperatures and temperature fluctuations which occurred in the principal parts of the cylinder walls, the breech-end, and the piston; because, after all, those were the relevant factors which determined the working of the engine. On those points they had at present no very definite information, and therefore one was likely to stumble in applying information such as was given in the Paper, as there were several missing links.

$\mathrm{He}$ would like to suggest that, although the Paper was very useful, it did not go quite in the particular direction where it would be most useful. Mr. Bickerton claimed on a previous occasion, and he understood Mr. Hiller had agreed with him, that the very small gas-engine was now a most reliable machine. He was disposed to agree with that view. When they had double-acting cylinders of 50 inches diameter (as they were now made), with walls of cast-iron 3 to 4 inches thick, the pistons being water-cooled and having very high piston-speeds, thermo-mechanical difficulties began to be serious, although not insuperable. It was just there that they wanted to know what were the temperatures in the walls, what were the fluctuations, and at what points were they likely to get serious cracks and overheating. If the authors would throw some light upon such problems, they would really advance the gasengine industry. It was possible to prosecute that class of researeh work without going to the expense of purchasing an engine costing $£ 10,000$ or $£ 12,000$. What they could do was to make a single cylinder of moderate size with very thick walls and pistons, put thermometers in and thermal-couples, and take measurements on those walls with various compressions and other conditions. Of course, the working conditions would not compare perfectly with those obtaining in large gas-engines, but still they would be much closer than at present with small experimental engines, and they would advance several steps towards obtaining information of vital value to the large gas-engine industry. If they could get something like definite information on points of that kind, engineers would 
feel justified in making alterations in designs which might lead to the great desideratum of the gas-engine industry-reduced first cost. A reduction of 10 per cent. on the first cost of a gas-engine was worth more than an addition of 5 per cent. on its thermal efficiency. He would not say that was true of every individual case, but he thought it was true of the general run of large gasengines. To be quite frank, the difficulty of selling large gasengines was not on the point of reliability so much as of cost. They were so reliable that several works were relying solely on large gas-engines. The most modern types, if properly looked after, were almost as reliable as a steam cotton-mill engine. The great dificulty was that in order to obtain this reliability, the engine was so costly that in many cases it did not pay to install it. Therefore the progress of the large gas-engine in this country was very slow, and was likely to be slow unless by some means the cost could be brought down very considerably. The first step towards reducing the cost was to find out the temperatures in the walls; secondly, to discover the influence of variable thicknesses ; and thirdly, to know the different qualities of metals in reducing the coefficient of expansion and the modulus of elasticity. When those points were solved, they would be able to make great progress. Till then he feared they would have to go very slowly indeed.

Mr. Danibl Adamson said that on page 376 the authors spoke of the "increased rate of transmission obtained in spite of a lower gas temperature," and said, "The reason is apparently to be found in the fact that the greater turbulence of the working fluid at the higher rates of speed increases its effective conductivity." There was no need for the authors to have inserted the word "apparently." An eminent predecessor of the Chairman of this Meeting, Professor Osborne Reynolds, studied the action of the principles of heat transmission many years ago, and the matter was very clearly put before them on many accasions by the late Dr. Nicolson in language (should he say) more suitable for consumption by the ordinary man in the street. Since then, 
(Mr. Daniel Adamson.)

almost every Paper dealing with the question of heat transmission had pointed in the same direction, not only in the design of steamgenerators, which was Dr. Nicolson's principal object in elaborating it,* but also in the design of electric generators it was found that the rate of cooling the parts of dynamos, which were subject to heating, varied directly as the rate at which the cooling air was passed over the surface. $\dagger$ They found that evening, as on many other occasions, the same fact brought to their notice. To put it in the authors' words, "it may be taken approximately that the effective conductivity was increased in the same ratio as the speed of the engine." He had Mr. Bickerton's authority in confirmation of that statement which he said was true. He would like once more to emphasize the great value of the work Dr. Nicolson did in that direction, and no doubt the authors would be able to confirm that remark.

Mr. J. Drummond Paton said there was one question he would like to see referred to, and that was the radiant-energy transmission capacity of gases. There was no mention in the Paper of the relative capacities of the gases which were the result of combustion.

With regard to Mr. Daniel Adamson's references to Dr. Nicolson's work, the results obtained by. Dr. Nicolson as to the transmission of heat from gases, he thought, went to prove that as they approached the velocity of transmission of radiant energy they increased the efficiency of heat production. The radiantenergy transmission was at the rate of something like 180,000 miles per second. The consequence was that if they got an immediate and open zone between the energized gas and the recipient body, they had radiant-energy transmission, and then again, where interception arose, linear velocity of the gases simply accelerated radiation.

* See his Paper before the Junior Inst. of Engineers, 14th Janugry 1909.

$\dagger$ See Symons and Walker's Paper, Inst. of Electrical Engineers, 25th January 1912 , Vol. 48 , page 674 . 
They ought to have some definition of the conditions under which the radiant effect was determined, and therefore located on cylinder walls. He referred particularly to the recent researches of Dr. Bone and the Bonecourt system. He thought they would find some solution of the transmission of radiant energy and the resultant product of heat if they actually analysed the radiant capacity of the various gases produced in combustion, and the zones in which they were formed.

Mr. Chiffond Digby said it would add to the value of the Paper very considerably if the indicator cards were published along with the other data on page 366. From the figures given in the Table it was evident there was some throttling effect before the inlet-valve, as the power was very much lower in proportion with 250 revolutions than with 200 . The maximum power as given even with the very rich mixture of $7: 1$ was much lower than was usual with an engine of that size. Under normal conditions, at 250 revolutions per minute the maximum power would be easily 44 b.h.p. If the light spring cards had been published, it would have been possible to ascertain directly whether the mechanical efficiency fall, which was very rapid at the higher speed, was due to that or not. In examining Table 2 (page 382) he noticed that the jacket-loss at 30 h.p. with a mixture of $7: 1$ was $19 \frac{1}{2}$ per cent. against $16 \cdot 1$ per cent. with the 9:1 mixture, which was approaching the best mixture for economy. This would point to slow burning of the charge with the rich mixture necessary to obtain the higher power under the existing conditions. He thought that if the cards were published, a much better opinion could be formed of the value of the figures at the higher speed than was possible at present.

Mr. W. J. WALkER, in the course of his reply, thanked the members for the way in which they had received the Paper and the various complimentary remarks made about it. The authors were well aware of the uncertainty attaching to the mechanical efficiency of a gas-engine, referred to by Professor Petavel, and in these trials 
(Mr. W. J. Walker.)

comparison was made between three different methods of obtaining the mechanical efficiency, to see how they agreed. These were the ratio of b.h.p. to i.h.p. method, the "radiation" method," and the "no load" method.* The first and the last agreed fairly well, but the radiation method gave values appreciably higher. Generally speaking, the average values of the first and last were taken. Professor Petavel also referred to the uncertainty of the value of the radiation loss. In the Paper, as stated, the radiation losses were taken as being the balancing quantities in each of the balance sheets. Some subsidiary tests, however, were also carried out according to the method outlined by Professor Hopkinson $\uparrow$ for the measurement of radiation losses, and these agreed sensibly with the results obtained by balance.

It was also pointed out that increase or decrease of jacketloss did not have a proportionate effect upon the efficiency. That was quite true. Dr. Dugald Clerk's Paper on "The Limits of Thermal Efficiency in Internal-Combustion Motors" $\ddagger$ first brought that point into prominence. At the same time that did not minimize the importance of the extent of these losses, since upon these depended the heat stresses set up in the jacket walls. This brought up a point referred to by Mr. Hiller (page 406) in connexion with efficiency and design, two factors which to some extent reacted upon one another and introduced the necessity of compromise. The efficiency of a gas-engine might at the most be increased by a small percentage, but the design of cylinder-jackets, more especially for large cylinders, was a question of greater importance than increase in efficiency. In the Paper the authors, with the desire to keep down an unwieldy mass of figures, had not given the divisions of heat flow in the various sections, namely, cooler, breech-end, barrel and exhaust gases, but no doubt these would be dealt with in a future Paper.

* Proceedings, Inst. C.E., 1906, vol. clxiii, prge 241.

+ Proceedings, I.Mech.E., 1908, page 417.

¥ Proceedings, Inst. C.E., 1907, vol. clxix, page 121. 
Mr. Hiller also referred to the desirability of research being carried on along the line of obtaining this increased reliability. He would like to point out, however, the difficulty which confronted those engaged in research in educational institutions. This difficulty was the one of obtaining apparatus which would be useful from both an educational and a research point of view. At the same time, he held that although it was not always possible to get the apparatus which was wanted, yet a good deal could be done with what was already installed.

Mr. Foster referred (page 411) to the mechanical efficiency of the engine decreasing so appreciably with speed, and expressed the opinion that that would not occur in engines which were designed to run at their own particular speed. With that opinion he (Mr: Walker) agreed to a certain extent. If an engine were designed to run at 200 revolutions per minute, then at 250 revolutions per minute its mechanical efficiency would be decreased by the increased resistance offered by the exhaust-pipe and valvepassages designed for a slower gas-speed. The pumping-losses would be increased. At the same time he thought that the mechanical efficiency of an engine designed to run at a higher speed would be decreased, although not nearly to the same extent as in the present case.

A question was raised with regard to the Table at the top of page 379. The engine used by Professor Hopkinson had a speed of 110 revolutions per minute, as compared with the 200 revolutions per minute of the Dundee engine. Personally, he did not think that the comparison would be appreciably affected; although the speed was lower, the relative figures would remain practically the same.

With reference to the statement on page 376 , which read, "it may be taken approximately that the effective conductivity is increased in the same ratio as the speed of the engine," it had been stated that that was an effect already obtained in experiments on the variation of heat transmission with gas velocity. Most experiments of this kind had been carried out on tubes of relatively small diameter compared to length, and did not conform to the case of a gas-engine 
(Mr. W. J. Walker.)

where there was a relatively large diameter. It was really a question as to whether convection or radiation was responsible for the larger share of the heat-loss. The work of Professor Hopkinson and W. T. David * on the radiation of gases showed that radiation was responsible for the loss of somewhere about 25 per cent. of the heat of combustion. How this proportion varied with ratio

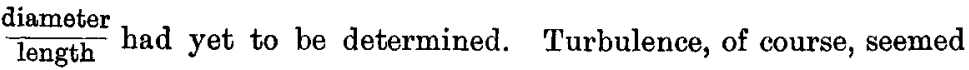
to indicate an increase in heat convection, but in view of the large proportion due to radiation, it seemed strange why convection should exert such an appreciable effect with increase of speed. He would suggest the following explanation. The proportion of heat radiated from the gas was unaltered by an increase in speed, but the passage of this radiated heat through the walls was facilitated by the increased scrubbing action of the gases at the higher speeds. The problem, however, was one to be solved by experiment. In conclusion, he desired to thank the Manchester members for their appreciation of the Paper and the interest they had shown in it.

Professor A. H. Gibson, who holds a Commission in the Royal Field Artillery, wrote expressing his regret at his unavoidable absence from the readings of the Paper and his appreciation of the way in which it was received both in London and Manchester. Owing to the strenuous work in camp he found that he had no time available to deal with the Discussion, to which he thought his co-author, Mr. Walker, had effectively replied.

* Phil. Trans. Roy. Soc., Vol. 211, page 375. 